\title{
Dynamical Behavior and Stability Analysis in a Hybrid Epidemiological-Economic Model with Incubation
}

\author{
Chao Liu, ${ }^{1,2}$ Wenquan Yue, ${ }^{3}$ and Peiyong Liu ${ }^{2,4}$ \\ ${ }^{1}$ Institute of Systems Science, Northeastern University, Shenyang 110004, China \\ ${ }^{2}$ State Key Laboratory of Integrated Automation of Process Industry, Northeastern University, Shenyang 110004, China \\ ${ }^{3}$ Changli Institute of Fruit Forestry, Hebei Academy of Agricultural and Forestry Sciences, Changli 066600, China \\ ${ }^{4}$ Institute of Biotechnology, College of Life and Health Sciences, Northeastern University, Shenyang 110004, China
}

Correspondence should be addressed to Chao Liu; singularsystem@163.com

Received 12 January 2014; Accepted 15 April 2014; Published 12 May 2014

Academic Editor: Weiming Wang

Copyright (C) 2014 Chao Liu et al. This is an open access article distributed under the Creative Commons Attribution License, which permits unrestricted use, distribution, and reproduction in any medium, provided the original work is properly cited.

\begin{abstract}
A hybrid SIR vector disease model with incubation is established, where susceptible host population satisfies the logistic equation and the recovered host individuals are commercially harvested. It is utilized to discuss the transmission mechanism of infectious disease and dynamical effect of commercial harvest on population dynamics. Positivity and permanence of solutions are analytically investigated. By choosing economic interest of commercial harvesting as a parameter, dynamical behavior and local stability of model system without time delay are studied. It reveals that there is a phenomenon of singularity induced bifurcation as well as local stability switch around interior equilibrium when economic interest increases through zero. State feedback controllers are designed to stabilize model system around the desired interior equilibria in the case of zero economic interest and positive economic interest, respectively. By analyzing corresponding characteristic equation of model system with time delay, local stability analysis around interior equilibrium is discussed due to variation of time delay. Hopf bifurcation occurs at the critical value of time delay and corresponding limit cycle is also observed. Furthermore, directions of Hopf bifurcation and stability of the bifurcating periodic solutions are studied. Numerical simulations are carried out to show consistency with theoretical analysis.
\end{abstract}

\section{Introduction}

In recent decades, plenty of mathematical models describing the population dynamics of infectious disease have been extensively utilized to understand the transmission mechanism of infectious disease within population ecosystem (see [1-4] and references therein). Much research efforts have been paid to susceptible-infective-recovered (SIR) vector disease model and corresponding model dynamics (see [5-12] and references therein). Generally, in modelling of communicable disease, the incidence rate (the rate of new infections) is considered to play a vital role in ensuring that the model can provide a reasonable qualitative description of the infectious disease dynamics $[3,4]$.

In order to discuss the spread of an infectious disease transmitted by a vector (e.g., mosquitoes and rats), Takeuchi et al. [7] formulated a delayed SIR epidemic model with a bilinear incidence rate. Beretta et al. [8] considered the global stability of disease free equilibrium and endemic equilibrium of model system; it was shown that the disease free equilibrium is globally stable for any time delay while the endemic equilibrium is not feasible. By constructing a suitable Lyapunov functional, sufficient conditions were derived to guarantee that if the endemic equilibrium is feasible, it is also globally stable for the delay being sufficiently small. Ruan and Wang [13] studied the global dynamics of an SIR model with vital dynamics and nonlinear incidence rate of saturated mass action and global qualitative and bifurcation analyses are carried out. Ma et al. [14] derived an explicit expression of lower bound of the infective individual of solution of model system, which was proposed as an open problem. They therefore gave an estimation of the length of time delay ensuring global asymptotic stability of the endemic equilibrium. $\mathrm{Xu}$ and $\mathrm{Ma}$ [15] proposed an SIR epidemic 
model with nonlinear incidence rate and time delay. By analyzing the corresponding characteristic equations, local stability of an endemic equilibrium and a disease free equilibrium are discussed. An SIR model with distributed delay and a general incidence function is studied in McCluskey [9], and the global dynamics for the SIR epidemiological system is analyzed in Zhou and Cui [10]. Wang et al. [11] considered the asymptotic behavior of the following SIR vector model:

$$
\begin{aligned}
& \dot{S}(t)=r\left(1-\frac{S(t)}{k}\right)-\beta S(t) I(t-\tau), \\
& \dot{I}(t)=\beta S(t) I(t-\tau)-\mu_{1} I(t)-m I(t), \\
& \dot{R}(t)=m I(t)-\mu_{2} R(t),
\end{aligned}
$$

where $S(t), I(t)$, and $R(t)$ represent the population density of susceptible, infective, and recovered host individuals at time $t$, respectively. It is assumed that the population growth of susceptible host individuals is governed by the logistic growth with a carrying capacity $k>0$ as well as intrinsic birth rate constant $r>0 . \beta>0$ is the average number of constants per infective per unit time and $\tau \geq 0$ denotes the incubation time, and $\mu_{1}>0$ and $\mu_{2}>0$ stand for the death rate of infective and recovered host individuals, respectively. $m>0$ represents the recovery rate of infective host individuals. The local stability of endemic equilibrium is investigated, and conditions for Hopf bifurcation to occur are derived in [11]. Along with the line of this research, Enatsu et al. [12] analyze stability of equilibria for a delayed SIR epidemic model, in which population growth is subject to logistic growth in absence of disease and the proposed model with a nonlinear incidence rate satisfying suitable monotonicity conditions.

Nowadays, biological resource within ecosystem is commercially harvested and sold with aim of achieving economic interest $[16,17]$. It is well known that harvesting has a strong impact on the dynamic evolution of population and several mathematical models have been established to discuss dynamic effects of harvest effort on population in ecological-epidemiological system, which can be found in [18-21] and the references therein. The role of harvesting in a predator-prey-parasite system is discussed in [18]; theoretical results show that, using impulsive harvesting effort as control parameter, it is not only possible to control the cyclic behavior of the system populations leading to the persistence of all species but other desired stable equilibrium including disease free can be obtained. A ratio-dependent eco-epidemiological system is proposed in [19] where prey population is subject to harvesting. Positive invariance, boundedness, stability of equilibria, and permanence of system have been established. In [20], an eco-epidemiological model is studied where prey disease is modeled by a susceptible-infective scheme, and the role of harvesting and switching on the dynamics of disease propagation and/or eradication is discussed. An eco-epidemiological model with distributed time delay and impulsive control strategy is investigated in [21]; local stability and complex dynamical behavior are discussed. Under the system of market economy, harvest effort is usually influenced by variation of economic interest of commercial harvesting $[16,22]$. It should be noted that the above mentioned related work [18-21] only concentrate on the role of harvest effort on population dynamics, while the dynamic effect of economic interest on commercial harvesting and indirect dynamic effect on ecosystem are not considered. The work done in [12] is an extension of [11] with nonlinear incidence rate, while dynamic effect of harvest effort on population dynamics is not considered.

Recently, some hybrid dynamical models are proposed in [23-28], which are utilized to discuss the interaction mechanism of harvested ecosystem from an economic perspective. Compared with the traditional mathematical models (differential equations or difference equations) discussing the population dynamics in ecosystem, the hybrid mathematical models proposed in [23-28] are made up of differential equations and algebraic equations, where differential equations concentrate on coexistence and interaction mechanism of population and algebraic equations offer a simpler way to study the effect of harvest effort on ecosystem from an economic perspective. Complex dynamical behavior and stability analysis in prey-predator ecosystems with stagestructured population and gestation delay are considered in [23-28]. In general, differential-algebraic models exhibit more complicated dynamics than ordinary differential models. The differential-algebraic models have been applied widely in power systems, aerospace engineering, chemical processes, social management systems, biological systems, network analysis and oil catalysis, and cracking process (see [29-31] and references therein). With the help of differentialalgebraic model for the power systems and bifurcation theory, complex dynamical behaviors of the power systems, especially the bifurcation phenomena that reveal the instability mechanism of power systems have been extensively studied, which can be found in [32-34] and the references therein. Furthermore, some applications of differentialalgebraic models in the field of economy, which can be found in $[35,36]$.

It is well known that the recovered host individuals are naturally immune to vector disease [1], and its potential economic interest can be commercially exploited. Furthermore, harvest effort is usually influenced by variation of economic interest of commercial harvesting $[16,22]$ under the system of market economy. Consequently, it is necessary to discuss the coexistence and interaction mechanism of population within harvested epidemiological ecosystem as well as dynamical effect of harvest effort due to variation of economic interest. However, as far as knowledge goes, nobody has explicitly proposed a mathematical model to discuss the dynamic effect of commercial harvest on epidemiological system under the system of market economy. The main objective of this paper is to investigate the transmission mechanism of infectious disease and dynamical effect of commercial harvest on population dynamics, especially the complex dynamical behavior and stability switch due to variation of incubation and commercial harvest economic interest. The organisation of the rest section of this paper is as follows. By introducing commercial harvest effort into model system (1), a hybrid epidemiological-economic model is established in Section 2. Positivity and permanence of solutions of model system are discussed in Section 3. In Section 4, qualitative analyses of 
model system are performed. Conditions for existence of interior equilibrium of model system are studied. Dynamical behavior of model system without incubation around the interior equilibrium is investigated due to variation of economic interest, and state feedback controllers are designed to stabilize model system around the desired interior equilibria. Furthermore, local stability analysis of model system with incubation is analyzed due to variation of time delay; directions of Hopf bifurcation and stability of the bifurcating periodic solutions are also studied. Numerical simulations are made in Section 5, which are utilized to support the theoretical findings obtained in this paper. Finally, this paper ends with a conclusion.

\section{Model Formulation}

In 1954, Gordon [22] proposed the economic theory of a common-property resource, which studies the effect of harvest effort on ecosystem from an economic perspective. In [22], an algebraic equation is proposed to investigate the economic interest of yield of the harvest effort, which takes form as follows:

\section{Net Economic Revenue (NER)}

$$
=\text { Total Revenue }(\mathrm{TR})-\text { Total Cost }(\mathrm{TC}) \text {. }
$$

Associated with model (1), an algebraic equation, which considers the economic interest $v$ of the harvest effort on recovered host individuals in epidemiological system, that is, $R(t)$, is established as follows:

$$
E(t)(w R(t)-c)=v
$$

where $E(t)$ represents the harvest effort on recovered host individuals at time $t . v$ represents the economic interest of harvest effort on the recovered host individuals. $w$ and $c$ represent unit price of harvested population and cost of harvest effort, respectively.

Based on (1) and (3), a delayed hybrid model which consists of three differential equations and an algebraic equation can be established as follows:

$$
\begin{aligned}
\dot{S}(t) & =r\left(1-\frac{S(t)}{k}\right)-\beta S(t) I(t-\tau), \\
\dot{I}(t) & =\beta S(t) I(t-\tau)-\mu_{1} I(t)-m I(t), \\
\dot{R}(t) & =m I(t)-\mu_{2} R(t)-E(t) R(t), \\
0 & =E(t)(w R(t)-c)-v,
\end{aligned}
$$

where $S(t), I(t), R(t), E(t)$, and other parameters share the same interpretations mentioned in (1) and (3), and initial conditions $\psi=\left(\psi_{1}, \psi_{2}, \psi_{3}, \psi_{4}\right)$ for model system (4) are defined in the Banach space:

$$
\begin{aligned}
\left\{\psi \in C^{+}\left([-\tau, 0], \mathbb{R}_{+}^{4}\right) \mid \psi_{1}(\theta)\right. & =S(\theta), \psi_{2}(\theta)=I(\theta), \\
\psi_{3}(\theta) & \left.=R(\theta), \psi_{4}(\theta)=E(\theta)\right\},
\end{aligned}
$$

where $\mathbb{R}_{+}^{4}=\left\{(S, I, R, E) \in \mathbb{R}^{4}: S \geq 0, I \geq 0, R \geq 0, E \geq 0\right\}$. It is also assumed that $\psi_{i}(0)>0(i=1,2,3,4)$ for a biological reason.

Model system (4) can be expressed in the following form:

$$
\Xi(t) \dot{X}(t)=F(X(t)),
$$

where

$$
\begin{aligned}
& X(t)=(S(t), I(t), R(t), E(t))^{T}, \\
& \Xi(t)=\left[\begin{array}{llll}
1 & 0 & 0 & 0 \\
0 & 1 & 0 & 0 \\
0 & 0 & 1 & 0 \\
0 & 0 & 0 & 0
\end{array}\right],
\end{aligned}
$$

$$
\begin{aligned}
F(X(t))= & {\left[\begin{array}{c}
F_{1}(X(t)) \\
F_{2}(X(t)) \\
F_{3}(X(t)) \\
F_{4}(X(t))
\end{array}\right] } \\
= & {\left[\begin{array}{c}
r\left(1-\frac{S(t)}{k}\right)-\beta S(t) I(t-\tau) \\
\beta S(t) I(t-\tau)-\mu_{1} I(t)-m I(t) \\
m I(t)-\mu_{2} R(t)-E(t) R(t) \\
E(t)(w R(t)-c)-v
\end{array}\right] . }
\end{aligned}
$$

Remark 1. The algebraic equation in model system (6) contains no differentiated variables; hence, the leading matrix $\Xi(t)$ in model system (6) has a corresponding zero row.

Remark 2. The model proposed in [11], which composed of differential equations, only discusses the interaction and coexistence mechanism of susceptible, infective, and recovered host individuals. Compared with the model proposed in [11], algebraic equations are incorporated into the model system (4), which focus on the economic interest of harvesting on recovered host individuals. Hence, the established model not only investigates interaction and coexistence mechanism of population in harvested ecosystem but also studies the dynamical behavior due to the variation of economic interest of commercial harvesting and incubation.

\section{Positivity and Permanence}

Theorem 3. Any solutions of model system (4) with initial conditions are positive.

Proof. For any solutions of model system (4), it is easy to show that $F_{i}: \mathbb{R}_{+}^{4+1} \rightarrow \mathbb{R}^{4}$ is locally Lipschitz and satisfies the condition, $\left.F_{i}(X(t))\right|_{X \in \mathbb{R}^{4}}>0$, where $F_{i}(X(t))(i=1,2,3,4)$ have been defined in model system (4).

Due to the lemma in [37] and Theorem A.4 in [38], any solution of the model system (4) with positive initial conditions exists uniquely and each component of the solution remains within the interval $\left[0, A_{0}\right)$ for some $A_{0}>0$. Standard and simple arguments show that solutions of model system (4) always exist and stay positive. Hence, this completes the positivity of the solutions of model system (4).

From a viewpoint of biological and economic interest perspective, persistence of solutions of model system (4) in 
the case of economic interest $v \geq 0$ will be investigated in this section. Some preliminaries are introduced as follows.

Definition 4 (see [39]). Model system (4) is said to be permanent if there exists a compact region $\Omega_{0} \in$ int $\Omega$ such that every solution of model system (4) with initial conditions will eventually enter and remain in region $\Omega_{0}$.

Definition 5 (see [39]). Consider a metric space $Q$ with metric $d$. The distance $d(x, y)$ of a point $x \in Q$ from a subset $Y$ of $Q$ is defined by

$$
d(x, y)=\inf _{y \in Y} d(x, y)
$$

It is further assumed that $Q$ is the closure of an open set $Q^{0}$, and $Q^{0}=\partial Q^{0}$ is nonempty and is the boundary of $Q^{0}$. Consequently, $Q^{0} \cup Q_{0}=Q, Q^{0} \cap Q_{0}=\varnothing$. We will also suppose that $T(t)$ is a $Q^{0}$ semigroup on $Q$ satisfying

$$
T(t): Q^{0} \longrightarrow Q^{0}, \quad T(t): Q_{0} \longrightarrow Q_{0} .
$$
$T_{\partial}(t)$.

Let $T_{\partial}(t)=\left.T(t)\right|_{Q_{0}}$ and $A_{\partial}$ be the global attractor for

Lemma 6 (see [39]). Suppose that $T(t)$ satisfies (9) and the following conditions hold.

(i) There is a $t_{0} \geq 0$ such that $T(t)$ is compact for $t>t_{0}$;

(ii) $T(t)$ is point dissipative in $Q$;

(iii) $\widetilde{A}_{\partial}=\bigcup_{x \in A_{\partial}} \omega(x)$ is isolated and has an acyclic covering $Z$.

Then $T(t)$ is uniformly persistent if and only if for each $Z_{i} \in Z$, $W^{s}\left(Z_{i}\right) \cap Q^{0}=\varnothing$ for $i=1,2, \ldots, n$.

Lemma 7 (see [40]). Consider the following equation:

$$
\dot{u}(t)=a u(t-\tau)-b u(t),
$$

where $a, b, \tau>0$ and $u(t)>0$ for all $-\tau \leq t \leq 0$; it derives the following:

(i) If $a<b$, then $\lim _{t \rightarrow+\infty} u(t)=0$,

(ii) If $a>b$, then $\lim _{t \rightarrow+\infty} u(t)=+\infty$.

Lemma 8. For any solutions of model system (4), we have

$$
\begin{gathered}
\limsup _{t \rightarrow+\infty} S(t) \leq k \\
\limsup _{t \rightarrow+\infty}(S(t)+I(t)) \leq \frac{k\left(r+\mu_{1}+m\right)^{2}}{4 r\left(\mu_{1}+m\right)} .
\end{gathered}
$$

Proof. By using Theorem 3, it follows from the first equation of model system (4) that

$$
\dot{S}(t) \leq r\left(1-\frac{S(t)}{k}\right) S(t)
$$

which derives that $\limsup _{t \rightarrow+\infty} S(t) \leq k$.
According to Theorem 3 and the first and second equation of model system (4), it gives that

$$
\dot{S}(t)+\dot{I}(t) \leq r\left(1-\frac{S(t)}{k}\right) S(t)-\left(\mu_{1}+m\right) I(t),
$$

which derives that $\limsup _{t \rightarrow+\infty}(S(t)+I(t)) \leq k\left(r+\mu_{1}+\right.$ $m)^{2} / 4 r\left(\mu_{1}+m\right)$.

Lemma 9. If $\mu_{1}+m<1$, then $(S(t), I(t))$ of solution of model system (4) with initial conditions satisfies

$$
\liminf _{t \rightarrow+\infty} S(t) \geq S_{\eta}, \quad \liminf _{t \rightarrow+\infty} I(t) \geq I_{\eta},
$$

where $S_{\eta}>0$ and $I_{\eta}>0$ are independent of corresponding initial values of model system (4).

Proof. Firstly, let $C^{+}\left([-\tau, 0], \mathbb{R}_{+}^{2}\right)$ denote space of continuous functions mapping $[-\tau, 0]$ into $\mathbb{R}_{+}^{2}$, where $\mathbb{R}_{+}^{2}=\{(x, y) \mid x \geq$ $0, y \geq 0\}$ :

$$
\begin{aligned}
Q_{1}= & \left\{\left(\phi_{1}, \phi_{2}\right) \in C^{+}\left([-\tau, 0], \mathbb{R}_{+}^{2}\right) \mid \phi_{1}(\theta)=0, \theta \in[-\tau, 0]\right\}, \\
Q_{2}= & \left\{\left(\phi_{1}, \phi_{2}\right) \in C^{+}\left([-\tau, 0], \mathbb{R}_{+}^{2}\right) \mid \phi_{1}(\theta)>0,\right. \\
& \left.\phi_{2}(\theta)=0, \theta \in[-\tau, 0]\right\} .
\end{aligned}
$$

Denote $Q_{0}=Q_{1} \cup Q_{2}, Q=C^{+}\left([-\tau, 0], \mathbb{R}_{+}^{2}\right)$ and $Q^{0}=$ int $C^{+}\left([-\tau, 0], \mathbb{R}_{+}^{2}\right)$.

Next, all conditions in Lemma 6 will be checked. In order to facilitate the proof, we consider the following subsystem of model system (4):

$$
\begin{aligned}
& \dot{S}(t)=r\left(1-\frac{S(t)}{k}\right)-\beta S(t) I(t-\tau), \\
& \dot{I}(t)=\beta S(t) I(t-\tau)-\mu_{1} I(t)-m I(t),
\end{aligned}
$$

where $S(\theta) \geq 0, I(\theta) \geq 0$ are continuous on $\theta \in[-\tau, 0]$ and $S(0)>0, I(0)>0$.

By Definition 5 and model system (16), it is easy to see that $Q^{0}$ and $Q_{0}$ are positively invariant, and conditions (i) and (ii) of Lemma 6 clearly hold.

Since model system (16) possesses two constant solutions in $Q_{0}: \widetilde{P}_{0} \in Q_{1}, \widetilde{P}_{1} \in Q_{2}$ with the following form:

$$
\begin{gathered}
\widetilde{P}_{0}=\left\{\left(\phi_{1}, \phi_{2}\right) \in C^{+}\left([-\tau, 0], \mathbb{R}_{+}^{2}\right) \mid \phi_{1}(\theta)=\phi_{2}(\theta)=0,\right. \\
\theta \in[-\tau, 0]\}, \\
\widetilde{P}_{1}=\left\{\left(\phi_{1}, \phi_{2}\right) \in C^{+}\left([-\tau, 0], \mathbb{R}_{+}^{2}\right) \mid \phi_{1}(\theta)=1, \phi_{2}(\theta)=0,\right. \\
\theta \in[-\tau, 0]\} .
\end{gathered}
$$

It follows from simple computation that

$$
\left.\dot{S}(t)\right|_{\left(\phi_{1}, \phi_{2}\right) \in Q_{1}}=0,\left.\quad S(t)\right|_{\left(\phi_{1}, \phi_{2}\right) \in Q_{1}}=0 \quad \text { for } t \geq 0 .
$$


Moreover, it follows from the second equation of model system (16) that

$$
\left.\dot{I}(t)\right|_{\left(\phi_{1}, \phi_{2}\right) \in Q_{1}}=-\left(\mu_{1}+m\right) I(t) \leq 0,
$$

which reveals that all points in $Q_{1}$ approach to $\widetilde{P}_{0}$; that is, $Q_{1}=W^{s}\left(\widetilde{P}_{0}\right)$. By using the similar analysis mentioned above, it can be also concluded that all points in $Q_{2}$ approach to $\widetilde{P}_{1}$; that is, $Q_{2}=W^{s}\left(\widetilde{P}_{1}\right)$. Based on the above analysis, it shows that invariant sets $\widetilde{P}_{0}$ and $\widetilde{P}_{1}$ are isolated invariant, and $\left\{\widetilde{P}_{0}, \widetilde{P}_{1}\right\}$ is isolated and an acyclic covering. It can be concluded that condition (iii) of Lemma 6 holds.

Finally, we will show that $W^{s}\left(\widetilde{P}_{i}\right) \cap Q^{0}=\varnothing$ for $i=0,1$. Based on the definition of $\widetilde{P}_{0}$, it is easy to show that $W^{s}\left(\widetilde{P}_{0}\right) \cap$ $Q^{0}=\varnothing$. We will show $W^{s}\left(\widetilde{P}_{1}\right) \cap Q^{0}=\varnothing$ in the following part.

If $W^{s}\left(\widetilde{P}_{1}\right) \cap Q^{0} \neq \varnothing$, then there exists a positive solution $(S(t), I(t))$ to model system (16) with $\lim _{t \rightarrow+\infty}(S(t), I(t))=$ $(1,0)$. If $\mu_{1}+m<1$, then $\mu_{1}+m<1-\epsilon$ holds for sufficiently small $\epsilon>0$ and there exists a positive constant $T=T(\epsilon)$ such that $S(t)>1-\epsilon>0$, and $0<I(t)<\epsilon$ for all $t \geq T$.

By the second equation of model system (16), it derives that

$$
\dot{I}(t) \geq(1-\epsilon) I(t-\tau)-\left(\mu_{1}+m\right) I(t)
$$

holds for all $t \geq T+\tau$.

Consider the following equation:

$$
\begin{aligned}
& \dot{x}(t)=(1-\epsilon) x(t-\tau)-\left(\mu_{1}+m\right) x(t), \quad t \geq T+\tau, \\
& x(t)=I(t), \quad T \leq t \leq T+\tau .
\end{aligned}
$$

Based on (21) and the comparison principle, it derives that $I(t) \geq x(t)$ for all $t>T$.

On the other hand, if $\mu_{1}+m<1$, then it follows from Lemma 7 that $\lim _{t \rightarrow+\infty} x(t)=+\infty$ for all solutions of (21). It can be concluded that $\lim _{t \rightarrow+\infty} I(t)=\infty$, which is a contradiction to $I(t)<\epsilon$. Consequently, it can be derived that $W^{s}\left(\widetilde{P}_{1}\right) \cap Q^{0}=\varnothing$.

According to the above analysis, all conditions of Lemma 6 hold. By using Lemma 6, it can be obtained that

$$
\liminf _{t \rightarrow+\infty} S(t) \geq S_{\eta}, \quad \liminf _{t \rightarrow+\infty} I(t) \geq I_{\eta},
$$

where $S_{\eta}>0$ and $I_{\eta}>0$ are independent of the corresponding initial values of model system (4).

Theorem 10. If $\mu_{1}+m<1, c \mu_{2}<m w I_{\eta}$, and $0 \leq v<$ $c \mu_{2}+m w I_{\eta}$, then all solutions of model system (4) with initial conditions are persistent.

Proof. According to Lemmas 8 and 9, it can be obtained that

$$
S_{\eta} \leq S(t) \leq k, \quad S(t)+I(t) \leq \frac{k\left(r+\mu_{1}+m\right)^{2}}{4 r\left(\mu_{1}+m\right)},
$$

hold for all $t>0$, which derive that

$$
I_{\eta} \leq I(t) \leq \frac{k\left(r+\mu_{1}+m\right)^{2}}{4 r\left(\mu_{1}+m\right)}-S_{\eta}
$$

When the economic interest $v=0$, it follows from Theorem 3 and the fourth equation of model system (4) that

$$
R(t)=\frac{c}{w}
$$

and $\dot{R}(t)=0$. Based on the third equation of model system (4), it can be computed that $E(t)=(m w / c) I(t)-\mu_{2}$. Accordng to (24), it derives that

$$
\begin{aligned}
0 & <\frac{m w I_{\eta}-c \mu_{2}}{c} \leq E(t) \\
& \leq \frac{m w\left[k\left(r+\mu_{1}+m\right)^{2}-4 S_{\eta} r\left(\mu_{1}+m\right)\right]}{4 c r\left(\mu_{1}+m\right)}-\mu_{2}
\end{aligned}
$$

provided that $c \mu_{2}<m w I_{\eta}$.

In the case of $v>0$, it derives that $E(t)=v /(w R(t)-c)$ based on implicit function theory [41]. According to the third equation of model system (4), it can be obtained that

$$
\dot{R}(t) \geq m I_{\eta}+\frac{c \mu_{2}-v}{w}-\mu_{2} R(t)
$$

which derives that

$$
\liminf _{t \rightarrow+\infty} R(t) \geq \frac{m w I_{\eta}+c \mu_{2}-v}{w \mu_{2}}:=\underline{R}>0
$$

provided that $0<v<c \mu_{2}+m w I_{\eta}$.

It follows from Theorem 3 and the third equation of model system (4) that

$$
\dot{R}(t) \leq m I(t)-\mu_{2} R(t),
$$

which derives that

$$
\limsup _{t \rightarrow+\infty} R(t) \leq \frac{m\left[k\left(r+\mu_{1}+m\right)^{2}-4 S_{\eta} r\left(\mu_{1}+m\right)\right]}{4 r \mu_{2}\left(\mu_{1}+m\right)}:=\bar{R} .
$$

Hence, it gives that $v /(w \bar{R}-c) \leq E(t) \leq v /(w \underline{R}-c)$, and it can be rewritten as follows:

$$
\begin{aligned}
& \frac{4 r \mu_{2} v\left(\mu_{1}+m\right)}{w m\left[k\left(r+\mu_{1}+m\right)^{2}-4 S_{\eta} r\left(\mu_{1}+m\right)\right]-4 r c \mu_{2}\left(\mu_{1}+m\right)} \\
& \leq E(t) \leq \frac{\mu_{2} v}{m w I_{\eta}-v} .
\end{aligned}
$$

Based on (23), (24), (25), and (26), it can be concluded that all solutions of model system (4) with initial conditions are persistent in the case of $v=0$, and it follows from (23), (24), (28), (30) and (31) that all solutions of model system (4) with initial conditions are persistent in the case of $0<v<$ $c \mu_{2}+m w I_{\eta}$. 


\section{Qualitative Analysis of Model System}

Dynamical effects of harvest effort and time delay on population dynamics are discussed in this section. It should be noted that the interior equilibrium biologically interprets that susceptible, infective, and recovered host individuals survive as well as harvest on recovered host individuals exists. Bifurcation phenomenon around the interior equilibria can reveal instability mechanism of model system, which are theoretically relevant to infectious disease control and sustainable yield on recovered host individuals in the real world. Consequently, we will mainly concentrate on dynamical behavior and local stability analysis around interior equilibrium of model system (4) in this paper.

4.1. Model System without Time Delay. In this section, dynamical behavior of model system (4) without time delay is investigated, and local stability analysis around the interior equilibrium is discussed due to variation of economic interest of commercial harvesting. Furthermore, state feedback controllers are designed to stabilize model system around the desired interior equilibria in the case of zero economic interest and positive economic interest, respectively.

\subsubsection{Singularity Induced Bifurcation}

Theorem 11. Model system (4) without time delay has a singularity induced bifurcation around the interior equilibrium, and $v=0$ is a bifurcation value. Furthermore, local stability switch occurs as $v$ increases through 0 .

Proof. Based on the economic theory of a common-property resource [22], there is a phenomenon of bioeconomic equilibrium in the case of zero harvest economic interest; that is, $v=0$. An interior equilibrium can be obtained as follows: $P^{*}\left(S^{*}, I^{*}, R^{*}, E^{*}\right)$, where $S^{*}=\left(\mu_{1}+m\right) / \beta, I^{*}=r(k \beta-$ $\left.\mu_{1}-m\right) / k \beta^{2}, R^{*}=c / w$, and $E^{*}=\left(w m r\left(k \beta-\mu_{1}-m\right)-\right.$ $\left.c k \mu_{2} \beta^{2}\right) / c k \beta^{2}$.

According to biological interpretation of the interior equilibrium, it follows that $S^{*}>0, I^{*}>0, R^{*}>0$ and $E^{*}>$ 0 . In order to guarantee the existence of interior equilibrium, some inequalities are satisfied:

$$
\begin{gathered}
k \beta-\mu_{1}-m>0, \\
w m r\left(k \beta-\mu_{1}-m\right)-c k \mu_{2} \beta^{2}>0 .
\end{gathered}
$$

Let $v$ be a bifurcation parameter, $H(t)=(S(t), I(t), R(t))^{T}$,

$$
\begin{aligned}
& h_{1}(H(t), E(t), v)=\left[\begin{array}{c}
r\left(1-\frac{S(t)}{k}\right)-\beta S(t) I(t-\tau), \\
\beta S(t) I(t-\tau)-\mu_{1} I(t)-m I(t), \\
m I(t)-\mu_{2} R(t)-E(t) R(t)
\end{array}\right], \\
& h_{2}(H(t), E(t), v)=E(t)(w R(t)-c)-v .
\end{aligned}
$$

It can be calculated that

$$
\begin{array}{r}
\left.\operatorname{trace}\left(D_{E} h_{1} \operatorname{adj}\left(D_{E} h_{2}\right)\left(D_{H} h_{2}, D_{E} h_{2}\right)\right)\right|_{P^{*}} \\
=-\frac{w m r\left(k \beta-\mu_{1}-m\right)-c k \mu_{2} \beta^{2}}{k \beta^{2}} .
\end{array}
$$

By virtue of (32), it can be obtained that

$$
\left.\operatorname{trace}\left(D_{E} h_{1} \operatorname{adj}\left(D_{E} h_{2}\right)\left(D_{H} h_{2}, D_{E} h_{2}\right)\right)\right|_{P^{*}} \neq 0 \text {. }
$$

Furthermore, it can be also calculated that

$$
\begin{aligned}
& \left|\begin{array}{ll}
D_{H} h_{1} & D_{E} h_{1} \\
D_{H} h_{2} & D_{E} h_{2}
\end{array}\right|_{P^{*}} \\
& =\frac{r\left(\mu_{1}+m\right)\left(k \beta-\mu_{1}-m\right)\left[w m r\left(k \beta-\mu_{1}-m\right)-c k \mu_{2} \beta^{2}\right]}{k^{2} \beta^{3}} .
\end{aligned}
$$

It follows from (32) that

$$
\left|\begin{array}{ll}
D_{H} h_{1} & D_{E} h_{1} \\
D_{H} h_{2} & D_{E} h_{2}
\end{array}\right|_{P^{*}} \neq 0 \text {. }
$$

Based on Section IV(A) in [42], $h_{3}(H(t), E(t), v)$ can be defined as follows:

$$
h_{3}(H(t), E(t), v)=\operatorname{det}\left(D_{E} g\right)=w R(t)-c
$$

By simple computing,

$$
\left|\begin{array}{lll}
D_{H} h_{1} & D_{E} h_{1} & D_{v} h_{1} \\
D_{H} h_{2} & D_{E} h_{2} & D_{v} h_{2} \\
D_{H} h_{3} & D_{E} h_{3} & D_{v} h_{3}
\end{array}\right|_{P^{*}}=\frac{c r\left(\mu_{1}+m\right)\left(k \beta-\mu_{1}+m\right)}{k \beta} .
$$

According to (32), it derives that

$$
\left|\begin{array}{lll}
D_{H} h_{1} & D_{E} h_{1} & D_{v} h_{1} \\
D_{H} h_{2} & D_{E} h_{2} & D_{v} h_{2} \\
D_{H} h_{3} & D_{E} h_{3} & D_{v} h_{3}
\end{array}\right|_{P^{*}} \neq 0
$$

Based on the above analysis, four items (i-iv) can be obtained as follows.

(i) It is easy to show that $D_{E} h_{2}$ has a simple zero eigenvalue:

$$
\left.h_{1}(H(t), E(t), v)\right|_{P^{*}}=0,\left.\quad h_{2}(H(t), E(t), v)\right|_{P^{*}}=0,
$$

and $\left.\operatorname{trace}\left(D_{E} h_{1} \operatorname{adj}\left(D_{E} h_{2}\right)\left(D_{H} h_{2}, D_{E} h_{2}\right)\right)\right|_{P^{*}} \neq 0$ based on (35).

(ii) It follows from (37) that $\left[\begin{array}{cc}D_{H} h_{1} & D_{E} h_{1} \\ D_{H} h_{2} & D_{E} h_{2}\end{array}\right]$ is nonsingular around $P^{*}$.

(iii) By virtue of (40), it can be shown that $\left[\begin{array}{ccc}D_{H} h_{1} & D_{E} h_{1} & D_{v} h_{1} \\ D_{H} h_{2} & D_{E} h_{2} & D_{v} h_{2} \\ D_{H} h_{3} & D_{E} h_{3} & D_{2} h_{3}\end{array}\right]$ is nonsingular around $P^{*}$; hence $\left[\begin{array}{lll}D_{H} h_{3} & D_{E} h_{3} & D_{v} h_{3}\end{array}\right]$ $\operatorname{rank}\left[\begin{array}{lll}D_{H} h_{1} & D_{E} h_{1} & D_{v} h_{1} \\ D_{H} h_{2} & D_{E} h_{2} & D_{v} h_{2} \\ D_{H} h_{3} & D_{E} h_{3} & D_{v} h_{3}\end{array}\right]=5$. 
(iv) It is easy to show rank $\left(h_{1}(H(t), E(t), v)\right)=3$ and $\operatorname{rank}\left(h_{2}(H(t), E(t), v)\right)=1$, which follows

$$
\begin{aligned}
\operatorname{rank} & {\left[\begin{array}{ccc}
D_{H} h_{1} & D_{E} h_{1} & D_{v} h_{1} \\
D_{H} h_{2} & D_{E} h_{2} & D_{v} h_{2} \\
D_{H} h_{3} & D_{E} h_{3} & D_{v} h_{3}
\end{array}\right] } \\
= & \operatorname{rank}\left(h_{1}(H(t), E(t), v)\right) \\
& +\operatorname{rank}\left(h_{2}(H(t), E(t), v)\right)+1 .
\end{aligned}
$$

It should be noted that the conditions for singularity induced bifurcation, which is introduced in Section III (A) in [42], consist of three conditions, that is, SI1, SI2, and SI3. According to the above items (i)-(iv), SI1, SI2, and SI3 are all satisfied; hence model (4) without time delay has a singularity induced bifurcation around the interior equilibrium $P^{*}$ and the bifurcation value is $v=0$.

Along with the line of the above proof, for model (4) without time delay, it follows from simple computing that

$$
\begin{aligned}
M & =-\left.\operatorname{trace}\left(D_{E} h_{1} \operatorname{adj}\left(D_{E} h_{2}\right)\left(D_{H} h_{2}, D_{E} h_{2}\right)\right)\right|_{P^{*}} \\
& =\frac{w m r\left(k \beta-\mu_{1}-m\right)-c k \mu_{2} \beta^{2}}{k \beta^{2}}, \\
N & =\left.\left[D_{v} h_{3}-\left[D_{H} h_{3}, D_{E} h_{3}\right]\left[\begin{array}{ll}
D_{H} h_{1} & D_{E} h_{1} \\
D_{H} h_{2} & D_{E} h_{2}
\end{array}\right]^{-1}\left[\begin{array}{l}
D_{v} h_{1} \\
D_{v} h_{2}
\end{array}\right]\right]\right|_{P^{*}} \\
& =\frac{c r\left(\mu_{1}+m\right)\left(k \beta-\mu_{1}-m\right)}{k \beta} .
\end{aligned}
$$

It follows from (32) that

$$
\frac{M}{N}=\frac{m r w\left(k \beta-\mu_{1}-m\right)-c k \mu_{2} \beta^{2}}{c r \beta\left(\mu_{1}+m\right)\left(k \beta-\mu_{1}-m\right)}>0 .
$$

Inequality (44) satisfies Theorem 3 of [42]. According to Theorem 3 of [42], when $v$ increases through 0 , one eigenvalue (denoted by $\lambda_{1}$ ) of model system (4) without time delay moves from $\mathbb{C}^{-}$to $\mathbb{C}^{+}$along the real axis by diverging through infinity; the movement behavior of this eigenvalue influences the stability of model system (4) without time delay.

Since the Jacobian of model system (4) without time delay evaluated around $P^{*}$ takes the following form:

$$
J_{P^{*}}=\left[\begin{array}{cccc}
-\frac{r S^{*}}{k} & -\beta S^{*} & 0 & 0 \\
\beta I^{*} & 0 & 0 & 0 \\
0 & m & -\left(\mu_{2}+E^{*}\right) & -R^{*} \\
0 & 0 & w E^{*} & 0
\end{array}\right] \text {, }
$$

according to the leading matrix $\Xi(t)$ in model system (4) and $J_{P^{*}}$, the characteristic equation of the model system (4) without time delay around $P^{*}$ is

$$
\operatorname{det}\left(\lambda \Xi-J_{P^{*}}\right)=0
$$

TABLE 1: Signs of real parts of eigen values of model (4) without time delay around interior equilibrium $P^{*}$.

\begin{tabular}{cccc}
\hline & $\operatorname{Re} \lambda_{1}$ & $\operatorname{Re} \lambda_{2}$ & $\operatorname{Re} \lambda_{3}$ \\
\hline$v<0$ & - & - & - \\
$v>0$ & + & - & - \\
\hline
\end{tabular}

By virtue of simple computation, the characteristic equation is as follows:

$$
\lambda^{2}+\frac{r S^{*}}{k} \lambda+\beta^{2} S^{*} I^{*}=0
$$

It can be concluded that the rest eigenvalues of model system (4) without time delay (denoted by $\lambda_{2}$ and $\lambda_{3}$ ) have negative real parts by using the Routh-Hurwitz criteria [43]. It follows from Theorem 3 in [42] that there is only one eigenvalue diverging to infinity as $v$ increases through 0 , and the rest eigenvalues are continuous and nonzero and cannot jump from one half open complex plane to another one as $v$ increases through 0 . It has been shown that $\lambda_{1}$ moves from $\mathbb{C}^{-}$to $\mathbb{C}^{+}$along the real axis by diverging through infinity. Therefore, $\lambda_{2}$ and $\lambda_{3}$ are continuous and bounded in the $\mathbb{C}^{-}$half plane as $v$ increases through 0 and their movement behaviors have no influence on the stability of model system (4) without time delay around the interior equilibrium $P^{*}$.

According to Table 1 and the stability theory, it can be concluded that model system (4) without time delay is stable around $P^{*}$ as $v<0$ and model system (4) without time delay is unstable around $P^{*}$ as $v>0$. Consequently, a stability switch occurs as $v$ increases through 0 .

Remark 12. Some preliminaries of singularity induced bifurcation are introduced below. Parameter dependent differential-algebraic hybrid system of the form

$$
\begin{array}{rlrl}
\dot{x}(t) & =h(x(t), y(t), \lambda), & h: R^{n} \times R^{m} \times R^{p} \longrightarrow R^{n}, \\
0 & =g(x(t), y(t), \lambda), \quad g: R^{n} \times R^{m} \times R^{p} \longrightarrow R^{m},
\end{array}
$$

where $x(t), y(t)$, and $\lambda$ have appropriate dimensions. It has been shown recently that there are generically three types of codimension one local bifurcation associated with the differential-algebraic model (48), namely, saddle-node bifurcation, Hopf bifurcation, and singularity induced bifurcation (see [42]).

The singularity induced bifurcation is firstly introduced and analyzed in $[42,44]$. It is a new type of bifurcation and does not occur in usual ordinary differential equation system, which has been characterized for differential-algebraic system, and later improved in $[45,46]$. Roughly speaking, the singularity induced bifurcation refers to a stability change of the differential-algebraic hybrid model (48) owing to some eigenvalues of related linearization $h_{x}-h_{y} g_{y}^{-1} g_{x}$ diverging to infinity when Jacobian $g_{y}$ is singular. 
One of the important consequences of the singularity induced bifurcation is that it leads to an impulse phenomenon, which may result in the collapse of the differentialalgebraic system (see [45]). More detailed introductions of the singularity induced bifurcation can be found in $[42,44-$ 46].

Remark 13. It follows from Theorem 11 that there is a phenomenon of singularity induced bifurcation around the interior equilibrium when economic interest increases through zero, which can cause local stability switch of model system (4). As stated in Remark 12, the singularity induced bifurcation can result in impulse phenomenon, which may lead to the collapse of the proposed model. In the harvested epidemiological-economic system, the impulse phenomenon is vividly reflected with the outbreak of infectious disease during a short period in the real world. Under this climate, the infected population will be beyond the carrying capacity of environment, which is disastrous for sustainable development of the harvested ecosystem as well as prosperous yield on recovered host individuals.

4.1.2. State Feedback Controller. In order to maintain the sustainable yield on recovered host individuals biological resource as well as economic interest of commercial harvesting at an ideal level, some corresponding control strategies should be taken to eliminate the impulse phenomenon caused by singularity induced bifurcation and stabilize model (4) without time delay. In this subsection, state feedback controllers are designed to stabilize model system (4) without time delay around corresponding interior equilibria in the case of $v=0$ and $v>0$, respectively.

According to the leading matrix $\Xi(t)$ in model system (4) and $J_{P^{*}}$ in (45) (the Jacobian of model system (4) without time delay around the interior equilibrium $P^{*}$ ), it can be calculated that $\operatorname{rank}\left(J_{P^{*}}, \Xi J_{P^{*}}, \Xi^{2} J_{P^{*}}, \Xi^{3} J_{P^{*}}\right)=4$. By using Theorem $2-2.1$ in [47], it is easy to show that the model system (4) without time delay is locally controllable around the interior equilibrium $P^{*}$ in the case of $v=0$. Consequently, a state feedback controller can be applied to stabilize the model system (4) without time delay around $P^{*}$. By using Theorem 3-1.2 in [47], a state feedback controller $u(t)=l\left(E(t)-E^{*}\right)$ ( $l$ is a feedback gain and $E^{*}$ is the component of the interior equilibrium $P^{*}$ ) can be applied to stabilize model system (4) without time delay around $P^{*}$.

Furthermore, the controlled model system (4) without time delay takes the following form:

$$
\begin{aligned}
\dot{S}(t) & =r\left(1-\frac{S(t)}{k}\right)-\beta S(t) I(t), \\
\dot{I}(t) & =\beta S(t) I(t)-\mu_{1} I(t)-m I(t), \\
\dot{R}(t) & =m I(t)-\mu_{2} R(t)-E(t) R(t), \\
0 & =E(t)(w R(t)-c)-v+u(t) .
\end{aligned}
$$

Theorem 14. When economic interest of harvesting is zero, $v=$ 0 , if the feedback gain $l$ satisfies the following inequality:

$$
\begin{gathered}
l>\max \left\{\frac{k w E^{*} R^{*}}{r S^{*}+k\left(\mu_{2}+E^{*}\right)}, \frac{w E^{*} R^{*}}{\mu_{2}+E^{*}}, \frac{r w E^{*} R^{*}}{k \beta^{2} I^{*}+r\left(\mu_{2}+E^{*}\right)},\right. \\
\left(w E^{*} R^{*}\left[2 k r\left(\mu_{2}+E^{*}\right)+2 k^{2} \beta^{2} I^{*}+r^{2} S^{*}\right]\right. \\
\left.+\sqrt{4 k^{4} \beta^{4} I^{* 2}+8\left(\mu_{2}+E^{*}\right) r k^{3} \beta^{2} I^{*}+r^{4} S^{* 2}}\right) \\
\times\left(2 r \left[r\left(\mu_{2}+E^{*}\right) S^{*}+k \beta^{2} I^{*} S^{*}\right.\right. \\
\left.\left.\left.+k\left(\mu_{2}+E^{*}\right)^{2}\right]\right)^{-1}\right\},
\end{gathered}
$$

then singularity induced bifurcation is eliminated and model system (49) is stable around $P^{*}$.

Proof. The Jacobian of the model system (49) evaluated at the interior equilibrium $P^{*}$ takes the form

$$
\widetilde{J}_{P^{*}}=\left[\begin{array}{cccc}
-\frac{r S^{*}}{k} & -\beta S^{*} & 0 & 0 \\
\beta I^{*} & 0 & 0 & 0 \\
0 & m & -\left(\mu_{2}+E^{*}\right) & -R^{*} \\
0 & 0 & w E^{*} & l
\end{array}\right] .
$$

According to the leading matrix $\Xi(t)$ in the model system (4) and $\widetilde{J}_{P^{*}}$, the characteristic equation of model system (49) around $P^{*}$ is $\operatorname{det}\left(\lambda \Xi-\widetilde{J}_{P^{*}}\right)=0$, which can be expressed as follows:

$$
\lambda^{3}+B_{1} \lambda^{2}+B_{2} \lambda+B_{3}=0
$$

where $B_{1}=\mu_{2}+E^{*}+r S^{*} / k-w E^{*} R^{*} / l, B_{2}=\beta^{2} I^{*} S^{*}+$ $\left(r S^{*} / k\right)\left(\mu_{2}+E^{*}-w E^{*} R^{*} / l\right)$, and $B_{3}=\beta^{2} I^{*} S^{*}\left(\mu_{2}+E^{*}-\right.$ $\left.w E^{*} R^{*} / l\right)$.

By using the Routh-Hurwitz criteria [43], model (49) is locally stable around $P^{*}$ if and only if $l$ satisfies

$$
\begin{gathered}
l>\max \left\{\frac{k w E^{*} R^{*}}{r S^{*}+k\left(\mu_{2}+E^{*}\right)}, \frac{w E^{*} R^{*}}{\mu_{2}+E^{*}}, \frac{r w E^{*} R^{*}}{k \beta^{2} I^{*}+r\left(\mu_{2}+E^{*}\right)},\right. \\
\left(w E^{*} R^{*}\left[2 k r\left(\mu_{2}+E^{*}\right)+2 k^{2} \beta^{2} I^{*}+r^{2} S^{*}\right]\right. \\
\left.+\sqrt{4 k^{4} \beta^{4} I^{* 2}+8\left(\mu_{2}+E^{*}\right) r k^{3} \beta^{2} I^{*}+r^{4} S^{* 2}}\right) \\
\times\left(2 r \left[r\left(\mu_{2}+E^{*}\right) S^{*}+k \beta^{2} I^{*} S^{*}\right.\right. \\
\left.\left.\left.+k\left(\mu_{2}+E^{*}\right)^{2}\right]\right)^{-1}\right\} .
\end{gathered}
$$

Consequently, if the feedback gain satisfies the above inequality, then model system (49) is stable around $P^{*}$ in the case of zero interest of harvesting. 
Let $\widetilde{P}^{*}\left(\widetilde{S}^{*}, \widetilde{I}^{*}, \widetilde{R}^{*}, \widetilde{E}^{*}\right)$ denote interior equilibrium of model (4) in the case of positive economic interest of harvesting $(v>0)$, where $\widetilde{S}^{*}=\left(\mu_{1}+m\right) / \beta, \widetilde{I}^{*}=r(k \beta-$ $\left.\mu_{1}-m\right) / k \beta^{2}, \widetilde{E}^{*}=v /\left(w R^{*}-c\right)$, and $\widetilde{R}^{*}$ satisfies the following equation:

$$
\widetilde{R}^{* 2}+\widetilde{B}_{2} \widetilde{R}^{*}+\widetilde{B}_{3}=0,
$$

where $\widetilde{B}_{2}=\left(k \beta^{2}\left(v-c \mu_{2}\right)+w m r\left(m+\mu_{1}-k \beta\right)\right) / k w \mu_{2} \beta^{2}$, $\widetilde{B}_{3}=c m r\left(k \beta-m u_{1}-m\right) / k w \mu_{2} \beta^{2}$.

Based on Routh-Hurwitz criteria [43], (54) has two positive roots if economic interest $v$ satisfies the following inequalities:

$$
\begin{aligned}
0<v<\min \left\{c \mu_{2}+\frac{w m r\left(k \beta-m-\mu_{1}\right)}{k \beta^{2}},\right. \\
\left.(1-c) c \mu_{2}+\frac{w m r(c+1)\left(k \beta-\mu_{1}-m\right)}{k \beta^{2}}\right\}
\end{aligned}
$$

$:=\widetilde{v}$

As analyzed above, there are two interior equilibria (denoted by $\widetilde{P}_{1}^{*}$ and $\widetilde{P}_{2}^{*}$ ) when $0<v^{*}<\widetilde{v}$. In this subsection, we only design the controller for the model (4) around the interior equilibrium $\widetilde{P}_{1}^{*}$. Some symmetric results about $\widetilde{P}_{2}^{*}$ can be also obtained, and $\widetilde{P}_{1}^{*}$ is denoted as $\widetilde{P}^{*}$ for simplicity in the following part.

Theorem 15. When economic interest of harvesting is positive, $0<v^{*}<\widetilde{v}$, if feedback gain $\tilde{l}$ of controller $u(t)=\widetilde{l}\left(E(t)-\widetilde{E}^{*}\right)$ satisfies following inequality:

$$
\begin{gathered}
\widetilde{l}>\max \left\{\frac{k w \widetilde{E}^{*} \widetilde{R}^{*}}{r \widetilde{S}^{*}+k\left(\mu_{2}+\widetilde{E}^{*}\right)}, \frac{w \widetilde{E}^{*} \widetilde{R}^{*}}{\mu_{2}+\widetilde{E}^{*}}, \frac{r w \widetilde{E}^{*} \widetilde{R}^{*}}{k \beta^{2} \widetilde{I}^{*}+r\left(\mu_{2}+\widetilde{E}^{*}\right)},\right. \\
\left(w \widetilde{E}^{*} \widetilde{R}^{*}\left[2 k r\left(\mu_{2}+\widetilde{E}^{*}\right)+2 k^{2} \beta^{2} \widetilde{I}^{*}+r^{2} \widetilde{S}^{*}\right]\right. \\
\left.+\sqrt{4 k^{4} \beta^{4} \widetilde{I}^{* 2}+8\left(\mu_{2}+\widetilde{E}^{*}\right) r k^{3} \beta^{2} \widetilde{I}^{*}+r^{4} \widetilde{S}^{* 2}}\right) \\
\times\left(2 r \left[r\left(\mu_{2}+\widetilde{E}^{*}\right) \widetilde{S}^{*}+k \beta^{2} \widetilde{I}^{*} \widetilde{S}^{*}\right.\right. \\
\left.\left.\left.+k\left(\mu_{2}+\widetilde{E}^{*}\right)^{2}\right]\right)\right\},
\end{gathered}
$$

then model system (49) is stable around the interior equilib$\operatorname{rium} \widetilde{P}^{*}\left(\widetilde{S}^{*}, \widetilde{I}^{*}, \widetilde{R}^{*}, \widetilde{E}^{*}\right)$.

Proof. The proof is similar to the proof of Theorem 14 of this paper.

Remark 16. It follows from (55) and Theorem 15 that economic interest of commercial harvesting should be regulated within certain interval $v \in(0, \widetilde{v})$, which guarantees the existence of interior equilibrium in the case of positive economic interest. After applying the state feedback controller into model system (4) without time delay, model system can be stabilized around the corresponding interior equilibrium, respectively. The elimination of the singularity induced bifurcation means the harvested epidemiologicaleconomic system restores to ecological balance and avoidance of infectious disease outbreak.

4.2. Model System with Time Delay. By analyzing corresponding characteristic equation of model system with time delay, local stability analysis around the interior equilibrium due to variation of time delay is discussed. Conditions for existence of Hopf bifurcation are studied. Furthermore, directions of Hopf bifurcation and stability of periodic solutions are investigated.

4.2.1. Local Stability and Hopf Bifurcation. As analyzed in the above subsection, in the case of time delay and positive economic interest of harvesting $0<v^{*}<\widetilde{v}$ where $\widetilde{v}$ is defined in (55), there are two interior equilibria $\widetilde{P}_{1}^{*}$ and $\widetilde{P}_{2}^{*}$ for model system (4) with respect to the positive economic interest $v^{*}$.

In this subsection, we only investigate dynamical behavior of model system (4) around the interior equilibrium $\widetilde{P}_{1}^{*}$. Some symmetric results about the interior equilibrium $\widetilde{P}_{2}^{*}$ can be also obtained, and $\widetilde{P}_{1}^{*}$ is denoted as $\widetilde{P}^{*}$ for simplicity. According to Jacobian evaluated at the interior equilibrium $\widetilde{P}^{*}$ and the leading matrix $\Xi(t)$ in model system (4), we can obtain the characteristic equation of model system (4) around $\widetilde{P}^{*}$, which can be expressed as follows:

$$
\begin{gathered}
\left|\begin{array}{cccr}
\lambda+\frac{r \widetilde{S}^{*}}{k} & \beta \widetilde{S}^{*} e^{-\lambda \tau} & 0 & 0 \\
-\beta \widetilde{I}^{*} & \lambda-\beta \widetilde{S}^{*} e^{-\lambda \tau}+\mu_{1}+m & 0 & 0 \\
0 & -m & \lambda+\mu_{1}+\widetilde{E}^{*} & \widetilde{R}^{*} \\
0 & 0 & -w \widetilde{E}^{*} & -\frac{v^{*}}{\widetilde{E}^{*}}
\end{array}\right|=0 \\
\Longrightarrow \\
M(\lambda)+N(\lambda) e^{-\lambda \tau}=0
\end{gathered}
$$

where

$$
\begin{aligned}
& M(\lambda)=\lambda^{3}+m_{1} \lambda^{2}+m_{2} \lambda+m_{3}, \\
& N(\lambda)=n_{1} \lambda^{2}+n_{2} \lambda+n_{3}, \\
& m_{1}=\mu_{2}+\left(\mu_{1}+m\right)\left(1+\frac{r}{k \beta}\right)-\frac{c v^{*}}{\left(w \widetilde{R}^{*}-c\right)^{2}}, \\
& m_{2}=\frac{\mu_{1}+m}{k \beta}\left[r\left(\mu_{1}+m+\mu_{2}-\frac{c v^{*}}{\left(w \widetilde{R}^{*}-c\right)^{2}}\right)\right. \\
&\left.\quad+k \beta\left(\mu_{2}-\frac{c v^{*}}{\left(w \widetilde{R}^{*}-c\right)^{2}}\right)\right],
\end{aligned}
$$




$$
\begin{aligned}
m_{3}= & \frac{r\left(\mu_{1}+m\right)^{2}}{k \beta}\left[\mu_{2}-\frac{c v^{*}}{\left(w \widetilde{R}^{*}-c\right)^{2}}\right], \\
n_{1}= & -\mu_{1}-m, \\
n_{2}= & -\left(\mu_{1}+m\right) \\
& \times\left[\mu_{2}+\frac{2 r\left(\mu_{1}+m\right)-k r \beta}{k \beta}-\frac{c v^{*}}{\left(w \widetilde{R}^{*}-c\right)^{2}}\right], \\
n_{3}= & -r\left(\mu_{1}+m\right)\left[\frac{2\left(\mu_{1}+m\right)}{k \beta}-1\right] \\
& \times\left[\mu_{2}-\frac{c v^{*}}{\left(w \widetilde{R}^{*}-c\right)^{2}}\right] .
\end{aligned}
$$

Now substituting $\lambda=i \sigma$ ( $\sigma$ is a positive real number) into (58) and separating the real and imaginary parts, two transcendental equations can be obtained as follows:

$$
\begin{gathered}
\sigma^{3}-m_{2} \sigma=n_{2} \sigma \cos (\sigma \tau)-\left(n_{3}-n_{1} \sigma^{2}\right) \sin (\sigma \tau), \\
m_{1} \sigma^{2}-m_{3}=\left(n_{3}-n_{1} \sigma^{2}\right) \cos (\sigma \tau)+n_{2} \sigma \sin (\sigma \tau) .
\end{gathered}
$$
that

By squaring and adding (60) and (61), it can be calculated

$$
\left(n_{3}-n_{1} \sigma^{2}\right)^{2}+n_{2}^{2} \sigma^{2}=\left(m_{1} \sigma^{2}-m_{3}\right)^{2}+\left(\sigma^{3}-m_{2} \sigma\right)^{2},
$$

$$
\sigma^{6}+C_{1} \sigma^{4}+C_{2} \sigma^{2}+C_{3}=0
$$

where $C_{1}=m_{1}^{2}-2 m_{2}-n_{1}^{2}, C_{2}=m_{2}^{2}-2 m_{1} m_{3}+2 n_{1} n_{3}-n_{2}^{2}, C_{3}=$ $m_{3}^{2}-n_{3}^{2}$.

According to the values of $C_{j},(j=1,2,3)$ and the RouthHurwitz criteria [43], a simple assumption that (58) has at least one positive real root $\sigma_{0}$ is $C_{3}<0$, which derives that $k \beta>3\left(\mu_{1}+m\right)$. Hence, under this assumption, (58) will have a pair of purely imaginary roots of the form $\pm i \sigma_{0}$.

By eliminating $\sin (\sigma \tau)$ from (60) and (61), it can be calculated that the $\tau_{j}^{*}$ corresponding to $\sigma_{0}$ is as follows:

$$
\begin{aligned}
\tau_{j}^{*}= & \frac{1}{\sigma_{0}} \\
& \times \arccos \left[\frac{\left.n_{2} \sigma_{0}^{2}\left(\sigma_{0}^{2}-m_{2}\right)+\left(n_{3}-n_{1} \sigma_{0}^{2}\right)\left(m_{1} \sigma_{0}^{2}-m_{3}\right)\right]}{\left(n_{3}-n_{1} \sigma_{0}^{2}\right)^{2}+\left(n_{2} \sigma_{0}\right)^{2}}\right] \\
& +\frac{2 j \pi}{\sigma_{0}},
\end{aligned}
$$

where $j=0,1,2, \ldots$.

By using Butler's lemma [48], model system (4) is locally stable around $\widetilde{P}^{*}$ for $\tau<\tau_{0}^{*}$. Subsequently, conditions for existence of Hopf bifurcation in [39] are utilized to investigate whether Hopf bifurcation occurs as $\tau$ increases through $\tau_{j}^{*}$.
Theorem 17. If $k \beta>3\left(\mu_{1}+m\right)$, then model system (4) undergoes Hopf bifurcation around the interior equilibrium $\widetilde{P}^{*}$ when $\tau=\tau_{j}^{*}, j=0,1,2, \ldots$ Furthermore, an attracting invariant closed curve bifurcates from interior equilibrium $\widetilde{P}^{*}$ when $\tau>\tau_{0}^{*}$ and $\left\|\tau-\tau_{0}^{*}\right\| \ll 1$.

Proof. As mentioned above, let $\lambda=i \sigma_{0}$ represent the purely imaginary root of (58). It follows from (58) that $\left|M\left(i \sigma_{0}\right)\right|=$ $\left|N\left(i \sigma_{0}\right)\right|$, which determines a set of possible values of $\sigma_{0}$.

In the following part, we determine the direction of motion of $\lambda=i \sigma_{0}$ as $\tau$ is varied; namely, we determine

$$
\Theta=\operatorname{sign}\left[\frac{\mathrm{d}(\operatorname{Re} \lambda)}{\mathrm{d} \tau}\right]_{\lambda=i \sigma_{0}}=\operatorname{sign}\left[\operatorname{Re}\left(\frac{\mathrm{d} \lambda}{\mathrm{d} \tau}\right)^{-1}\right]_{\lambda=i \sigma_{0}} .
$$

By differentiating (58) with respect to $\tau$, it can be obtained that

$$
\begin{aligned}
\left(\frac{\mathrm{d} \lambda}{\mathrm{d} \tau}\right)^{-1}= & \frac{3 \lambda^{2}+2 m_{1} \lambda+m_{2}}{\lambda e^{-\lambda \tau}\left(n_{1} \lambda^{2}+n_{2} \lambda+n_{3}\right)}+\frac{2 n_{1} \lambda+n_{2}}{\lambda\left(n_{1} \lambda^{2}+n_{2} \lambda+n_{3}\right)} \\
& -\frac{\tau}{\lambda} \\
= & \frac{3 \lambda^{2}+2 m_{1} \lambda+m_{2}}{-\lambda\left(\lambda^{3}+m_{1} \lambda^{2}+m_{2} \lambda+m_{3}\right)} \\
& +\frac{2 n_{1} \lambda+n_{2}}{\lambda\left(n_{1} \lambda^{2}+n_{2} \lambda+n_{3}\right)}-\frac{\tau}{\lambda} \\
= & \frac{2 \lambda^{3}+m_{1} \lambda^{2}-m_{3}}{-\lambda^{2}\left(\lambda^{3}+m_{1} \lambda^{2}+m_{2} \lambda+m_{3}\right)} \\
& +\frac{n_{1} \lambda^{2}-n_{3}}{\lambda^{2}\left(n_{1} \lambda^{2}+n_{2} \lambda+n_{3}\right)}-\frac{\tau}{\lambda} .
\end{aligned}
$$

From (62) and the above equation, it can be obtained that

$$
\begin{gathered}
\Theta=\operatorname{sign}\left[\operatorname{Re}\left(\frac{\mathrm{d} \lambda}{\mathrm{d} \tau}\right)^{-1}\right]_{\lambda=i \sigma_{0}} \\
=\frac{1}{\sigma_{0}^{2}} \operatorname{sign}\left[\frac{\left(m_{3}+m_{1} \sigma_{0}^{2}\right)\left(m_{1} \sigma_{0}^{2}-m_{3}\right)+2 \sigma_{0}^{4}\left(\sigma_{0}^{2}-m_{2}\right)}{\left(m_{1} \sigma_{0}^{2}-m_{3}\right)^{2}+\left(\sigma_{0}^{3}-m_{2} \sigma_{0}\right)^{2}}\right. \\
\left.+\frac{\left.\left(n_{1} \sigma_{0}^{2}+n_{3}\right)\left(n_{3}-n_{1} \sigma_{0}^{2}\right)\right]}{\left(n_{3}-n_{1} \sigma_{0}^{2}\right)^{2}+\left(n_{2} \sigma_{0}\right)^{2}}\right] \\
=\operatorname{sign}\left[\left(\left(m_{3}+m_{1} \sigma_{0}^{2}\right)\left(m_{1} \sigma_{0}^{2}-m_{3}\right)+2 \sigma_{0}^{4}\left(\sigma_{0}^{2}-m_{2}\right)\right.\right. \\
\left.\quad+\left(n_{1} \sigma_{0}^{2}+n_{3}\right)\left(n_{3}-n_{1} \sigma_{0}^{2}\right)\right)
\end{gathered}
$$




$$
\begin{aligned}
& \left.\times\left(\left(m_{1} \sigma_{0}^{2}-m_{3}\right)^{2}+\left(\sigma_{0}^{3}-m_{2} \sigma_{0}\right)^{2}\right)^{-1}\right] \\
= & \operatorname{sign}\left[\frac{2 \sigma_{0}^{6}+\left(m_{1}^{2}-2 m_{2}-n_{1}^{2}\right) \sigma_{0}^{4}+n_{3}^{2}-m_{3}^{2}}{\left(m_{1} \sigma_{0}^{2}-m_{3}\right)^{2}+\left(\sigma_{0}^{3}-m_{2} \sigma_{0}\right)^{2}}\right], \\
= & \operatorname{sign}\left[\frac{2 \sigma_{0}^{6}+C_{1} \sigma_{0}^{4}-C_{3}}{\left(m_{1} \sigma_{0}^{2}-m_{3}\right)^{2}+\left(\sigma_{0}^{3}-m_{2} \sigma_{0}\right)^{2}}\right] .
\end{aligned}
$$

According to the values of $C_{j},(j=1,2,3)$ given in (58) of this paper, it is easy to show that $C_{1}=m_{1}^{2}-2 m_{2}-n_{1}^{2}=$ $r^{2}\left(\mu_{1}+m\right)^{2} / k^{2} \beta^{2}+\left[\mu_{2}-c v^{*} /(w \widetilde{R}-c)^{2}\right]^{2}>0$.

Furthermore, if $k \beta>3\left(\mu_{1}+m\right)$, then it can be shown that $C_{3}>0$. Hence, it can be concluded that $2 \sigma_{0}^{6}+C_{1} \sigma_{0}^{4}-$ $C_{3}>0$, which derives $\operatorname{sign}[\mathrm{d}(\operatorname{Re} \lambda) / \mathrm{d} \tau]_{\tau=\tau_{j}^{*}, \sigma=\sigma_{0}}>0$. Consequently, the transversality condition holds and Hopf bifurcation occurs at $\sigma=\sigma_{0}, \tau=\tau_{j}^{*}$. Furthermore, an attracting invariant closed curve bifurcates from interior equilibrium $\widetilde{P}^{*}$ when $\tau>\tau_{0}^{*}$ and $\left\|\tau-\tau_{0}^{*}\right\| \ll 1$.

Remark 18. $\operatorname{sign}[\mathrm{d}(\operatorname{Re} \lambda) / \mathrm{d} \tau]_{\tau=\tau_{j}^{*}}>0$ signifies that there exists at least one eigenvalue with positive real part for $\tau=$ $\tau_{j}^{*}$, and the conditions for Hopf bifurcation in [39] are also satisfied yielding the required periodic solution.

4.2.2. Properties of Hopf Bifurcation. By using normal theory and center manifold theorem [49], directions of Hopf bifurcation and stability of the bifurcating periodic solutions are discussed in this section. As analyzed in Section 4.1.2, when economic interest of harvesting $0<v^{*}<\widetilde{v}(\bar{v}$ is defined in (55)), it follows from implicit function theorem [41] and the fourth equation of model system (4) that $E(t)=v^{*} /(w R(t)-$ $c)$. Furthermore, model system (4) can be transformed into the following form:

$$
\begin{aligned}
& \dot{S}(t)=r\left(1-\frac{S(t)}{k}\right)-\beta S(t) I(t-\tau), \\
& \dot{I}(t)=\beta S(t) I(t-\tau)-\mu_{1} I(t)-m I(t), \\
& \dot{R}(t)=m I(t)-\mu_{2} R(t)-\frac{v^{*} R(t)}{w R(t)-c} .
\end{aligned}
$$

Firstly, some transformations associated with component $\left(\widetilde{S}^{*}, \widetilde{I}^{*}, \widetilde{R}^{*}\right)$ of interior equilibrium $\widetilde{P}^{*}$ are given as follows:

$$
\begin{gathered}
y_{1}=S-\widetilde{S}^{*}, \quad y_{2}=I-\widetilde{I}^{*}, \quad y_{3}=R-\widetilde{R}^{*}, \\
\bar{y}_{i}(t)=y_{i}(\tau t), \quad \tau=\rho+\tau_{j}, \rho \in \mathbb{R}=(-\infty,+\infty) .
\end{gathered}
$$

Then $\rho=0$ is the Hopf bifurcation value of model system (4). Bars of variables are dropped for simplicity of notations; model system (4) is transformed to a functional differential equation in $C=C\left([-1,0], \mathbb{R}^{3}\right)$ as

$$
\dot{y}(t)=L_{\rho}\left(y_{t}\right)+f\left(\rho, y_{t}\right),
$$

where $C=C\left([-1,0], \mathbb{R}^{3}\right)$ is the Banach space of continuous functions mapping the interval $[-\tau, 0]$ into $\mathbb{R}^{3}, y(t)=$ $\left(y_{1}(t), y_{2}(t), y_{3}(t)\right)^{T} \in \mathbb{R}^{3}, y_{t}(\theta)=y(t+\theta)$ for $\theta \in[-\tau, 0]$ and $L_{\rho}: C \rightarrow \mathbb{R}^{3}, f: \mathbb{R} \times C \rightarrow \mathbb{R}^{3}$ are defined as follows, respectively:

$$
\begin{aligned}
L_{\rho}(\phi)= & \left(\tau_{j}+\rho\right) \\
& \times\left(\begin{array}{ccc}
-\frac{r \widetilde{S}^{*}}{k} & 0 & 0 \\
\beta \widetilde{I}^{*} & -\left(\mu_{1}+m\right) & 0 \\
0 & m & -\mu_{2}+\frac{c v^{*}}{\left(w \widetilde{R}^{*}-c\right)^{2}}
\end{array}\right) \\
& \times\left(\begin{array}{c}
\phi_{1}(0) \\
\phi_{2}(0) \\
\phi_{3}(0)
\end{array}\right)+\left(\tau_{j}+\rho\right)\left(\begin{array}{ccc}
0 & -\beta \widetilde{S}^{*} & 0 \\
0 & \beta \widetilde{S}^{*} & 0 \\
0 & 0 & 0
\end{array}\right) \\
& \times\left(\begin{array}{c}
\phi_{1}(-1) \\
\phi_{2}(-1) \\
\phi_{3}(-1)
\end{array}\right), \\
f(\rho, \phi)= & \left(\tau_{j}+\rho\right)\left(\begin{array}{c}
-\frac{r}{k} \phi_{1}^{2}(0)-\beta \phi_{1}(0) \phi_{2}(-1) \\
\beta \phi_{1}(0) \phi_{2}(-1) \\
\sum_{j=1}^{\infty} f_{j}^{(3)} \phi_{3}^{j}(0)
\end{array}\right), \quad(72)
\end{aligned}
$$

where $f_{j}^{(3)}=\left.\left(\mathrm{d}^{i}\left(-v^{*} R /(w R-c)\right) / \mathrm{d} R^{i}\right)\right|_{\left.\tilde{S}^{*}, \tilde{I}^{*}, \widetilde{R}^{*}\right)}, j=1,2,3$.

It is easy to show that $L_{\rho}$ is a continuous linear function mapping $C$ into $\mathbb{R}^{3}$. According to Riesz representation theorem [40], there exists a $3 \times 3$ matrix function $\eta(\theta, \rho)$ of bounded variation for $\theta \in[-1,0]$ such that

$$
L_{\rho}(\phi)=\int_{-1}^{0} \mathrm{~d} \eta(\theta, \rho) \phi(\theta),
$$

where $\phi \in C\left([-1,0], \mathbb{R}^{3}\right)$.

In fact, we can choose

$$
\begin{aligned}
\eta(\theta, \rho)= & \left(\tau_{j}+\rho\right) \\
& \times\left(\begin{array}{ccc}
-\frac{r \widetilde{S}^{*}}{k} & 0 & 0 \\
\beta \widetilde{I}^{*} & -\left(\mu_{1}+m\right) & 0 \\
0 & m & -\mu_{2}+\frac{c v^{*}}{\left(w \widetilde{R}^{*}-c\right)^{2}}
\end{array}\right) \\
& \times \delta(\theta) \\
& -\left(\tau_{j}+\rho\right)\left(\begin{array}{ccc}
0 & -\beta \widetilde{S}^{*} & 0 \\
0 & \beta \widetilde{S}^{*} & 0 \\
0 & 0 & 0
\end{array}\right) \delta(\theta+1),
\end{aligned}
$$

where $\delta$ denotes the Dirac delta function. 
If $\phi$ is any given function in $C\left([-1,0], \mathbb{R}^{3}\right)$ and $y(\phi)$ is the unique solution of the linearized equation $\dot{y}(t)=L_{\rho}\left(y_{t}\right)$ of (70) with initial function $\phi$ at zero, then the solution operator $\widetilde{T}(t): C \rightarrow C$ is defined by

$$
\widetilde{T}(t) \phi=y_{t}(\phi), \quad t \geq 0 .
$$

It follows from Lemma 7.1.1 in [39] that $\widetilde{T}(t), t \geq 0$ is a strongly continuous semigroup of linear transformation on $[0,+\infty)$ and the infinitesimal generator $A_{\rho}$ of $\widetilde{T}(t), t \geq 0$ is as follows:

$$
A_{\rho}(\phi)= \begin{cases}\frac{\mathrm{d} \phi(\theta)}{\mathrm{d} \theta}, & \theta \in[-1,0) \\ \int_{-1}^{0} \mathrm{~d} \eta(\rho, s) \phi(s), & \theta=0,\end{cases}
$$

for $\phi \in C^{1}\left([-1,0], \mathbb{R}^{3}\right)$, the space of functions mapping the interval $[-1,0]$ into $\mathbb{R}^{3}$ which have continuous first derivative and also define

$$
R(\rho)(\phi)= \begin{cases}0, & \theta \in[-1,0) \\ f(\rho, \phi), & \theta=0\end{cases}
$$

then model system (70) is equivalent to

$$
\dot{y}_{t}=A(\rho) y_{t}+R(\rho) y_{t} \text {. }
$$

For $\psi \in C^{1}\left([0,1],\left(\mathbb{R}^{3}\right)^{*}\right)$, the space of functions mapping interval $[0,1]$ into the three-dimensional row vectors which have continuous first derivative, define

$$
A^{*} \phi(s)= \begin{cases}-\frac{\mathrm{d} \psi(s)}{\mathrm{d} s}, & s \in(0,1] \\ \int_{-1}^{0} \mathrm{~d} \eta^{T}(t, 0) \psi(-t), & s=0,\end{cases}
$$

and a bilinear inner product

$$
\begin{aligned}
\langle\psi(s), \phi(\theta)\rangle= & \bar{\psi}(0) \phi(0) \\
& -\int_{-1}^{0} \int_{\xi=0}^{\theta} \bar{\psi}(\xi-\theta) \mathrm{d} \eta(\theta) \phi(\xi) \mathrm{d} \xi,
\end{aligned}
$$

where $\eta(\theta)=\eta(\theta, 0)$. It follows from the above analysis $A(0)$ and $A^{*}$ are adjoint operators.

By virtue of discussion in Section 4.2.1, $\pm i \omega_{0} \tau_{j}$ are eigenvalues of $A(0)$. Hence, they are also eigenvalues of $A^{*}$. In the following, eigenvectors of $A(0)$ and $A^{*}$ are corresponding to $i \omega_{0} \tau_{j}$ and $-i \omega_{0} \tau_{j}$, respectively.

Suppose $q(\theta)=(1, a, b)^{T} e^{i \omega_{0} \tau_{j} \theta}$ is the eigenvectors of $A(0)$ corresponding to $i \omega_{0} \tau_{j}$, which derives that
$A(0) q(\theta)=i \omega_{0} \tau_{j} q(\theta)$. By using the definition of $A(0),(71)$ and (72), it gives that

$$
\begin{gathered}
\left(\begin{array}{ccc}
-\frac{r \widetilde{S}^{*}}{k} & 0 & 0 \\
\beta \widetilde{I}^{*} & -\left(\mu_{1}+m\right) & 0 \\
0 & m & -\mu_{2}+\frac{c v^{*}}{\left(w \widetilde{R}^{*}-c\right)^{2}}
\end{array}\right) q(0) \\
+\left(\begin{array}{ccc}
0 & -\beta \widetilde{S}^{*} & 0 \\
0 & \beta \widetilde{S}^{*} & 0 \\
0 & 0 & 0
\end{array}\right) q(-1)=i \omega_{0} q(0) .
\end{gathered}
$$

For $q(-1)=q(0) e^{-i \omega_{0} \tau_{j}}$, then it can be obtained that

$$
\begin{aligned}
& a=-\frac{r\left(\mu_{1}+m\right)+i k \beta \omega_{0}}{k \beta\left(\mu_{1}+m\right) e^{-i \omega_{0} \tau_{j}}}, \\
& b=\frac{m\left(w \widetilde{R}^{*}-c\right)^{2}\left[r\left(m+\mu_{1}\right)+i k \beta \omega_{0}\right]}{k \beta\left(\mu_{1}+m\right)\left[c v^{*}-\left(\mu_{2}+i \omega_{0}\right)\left(w \widetilde{R}^{*}-c\right)^{2}\right] e^{-i \omega_{0} \tau_{j}}} .
\end{aligned}
$$

Similarly, it follows from simple computation that eigenvector $q^{*}(s)=J\left(1, a^{*}, b^{*}\right) e^{i \omega_{0} \tau_{j} s}$ of $A^{*}$ is corresponding to $-i \omega_{0} \tau_{j}$, where

$$
\begin{aligned}
& a^{*}=-\frac{r\left(\mu_{1}+m\right)-i k \beta \omega_{0}}{k \beta\left(\mu_{1}+m\right) e^{i \omega_{0} \tau_{j}},} \\
& b^{*}=\frac{m\left(w \widetilde{R}^{*}-c\right)^{2}\left[r\left(\mu_{1}+m\right)-i k \beta \omega_{0}\right]}{k \beta\left(\mu_{1}+m\right)\left[c v^{*}-\left(\mu_{2}-i \omega_{0}\right)\left(w \widetilde{R}^{*}-c\right)^{2}\right] e^{i \omega_{0} \tau_{j}}} .
\end{aligned}
$$

In order to assume $\left\langle q^{*}(s), q(\theta)\right\rangle=1$, we need to determine the value of $J$ in the following part.

By virtue of (80), it derives that

$$
\begin{aligned}
& \left\langle q^{*}(s), q(\theta)\right\rangle \\
& =\bar{J}\left(1, \bar{a}^{*}, \bar{b}^{*}\right)(1, a, b)^{T} \\
& -\int_{-1}^{0} \int_{\xi=0}^{\theta} \bar{J}\left(1, a^{*}, b^{*}\right) e^{-i \omega_{0} \tau_{j}(\xi-\theta)} \mathrm{d} \eta(\theta) \\
& \quad \times(1, a, b)^{T} e^{i \omega_{0} \tau_{j} \xi} \mathrm{d} \xi \\
& =\bar{J}\left[1+a \bar{a}^{*}+b \bar{b}^{*}\right. \\
& \left.\quad-\int_{-1}^{0}\left(1, \bar{a}^{*}, \bar{b}^{*}\right) \theta e^{i \omega_{0} \tau_{j} \theta} \mathrm{d} \eta(\theta)(1, a, b)^{T}\right] \\
& =\bar{J}\left[1+a \bar{a}^{*}+b \bar{b}^{*}+k \bar{b}^{*} \tau_{j}\left(\widetilde{R}^{*}+b \widetilde{S}^{*}\right) e^{i \omega_{0} \tau_{j}}\right] .
\end{aligned}
$$

Hence, we can choose $J$ as follows:

$$
J=\frac{1}{1+\bar{a} a^{*}+\bar{b} b^{*}+k b^{*} \tau_{j}\left(\widetilde{R}^{*}+b \widetilde{S}^{*}\right) e^{i \omega_{0} \tau_{j}} .}
$$


Next, we will compute the coordinate to describe the centre manifold $C_{0}$ at $\rho=0$. Let $y_{t}$ be the solution of (78) when $\rho=0$.

Define

$z(t)=\left\langle q^{*}, y_{t}\right\rangle, \quad W(t, \theta)=y_{t}(\theta)-2 \operatorname{Re}\{z(t) q(\theta)\}$.

On the center manifold $C_{0}$, it derives that

$$
W(t, \theta)=W(z(t), \bar{z}(t), \theta)
$$

where

$$
W(z(t), \bar{z}(t), \theta)=W_{20}(\theta) \frac{z^{2}}{2}+W_{11}(\theta) z \bar{z}+W_{02} \frac{\bar{z}^{2}}{2}+\cdots
$$

$z$ and $\bar{z}$ are local coordinates for center manifold $C_{0}$ in the direction of $q^{*}$ and $\bar{q}^{*}$.

It is noted that $W$ is real if $y_{t}$ is real, and we only consider real solutions. For solution $y_{t} \in C_{0}$ of (78), since $\rho=0$, it derives that

$$
\begin{aligned}
\dot{z}(t) & =i \omega_{0} \tau_{j} z+\bar{q}^{*}(0) f(0, W(z, \bar{z}, 0)+2 \operatorname{Re}\{z q(\theta)\}) \\
& \triangleq i \omega_{0} \tau_{j} z+\bar{q}^{*}(0) f_{0}(z, \bar{z})
\end{aligned}
$$

The above equation can be rewritten as follows:

$$
\dot{z}(t)=i \omega_{0} \tau_{j} z(t)+g(z, \bar{z}),
$$

where

$$
\begin{aligned}
g(z, \bar{z}) & =\bar{q}^{*}(0) f_{0}(z, \bar{z}) \\
& =g_{20} \frac{z^{2}}{2}+g_{11} z \bar{z}+g_{02} \frac{\bar{z}^{2}}{2}+g_{21} \frac{z^{2} \bar{z}}{2}+\cdots
\end{aligned}
$$

It follows from (86) and (88) that

$$
\begin{aligned}
y_{t}(\theta)= & W(t, \theta)+2 \operatorname{Re}\{z(t) q(\theta)\} \\
= & W_{20}(\theta) \frac{z^{2}}{2}+W_{11}(\theta) z \bar{z}+W_{02}(\theta) \frac{\bar{z}^{2}}{2} \\
& +(1, a, b)^{T} e^{i \omega_{0} \tau_{j} \theta} z+(1, \bar{a}, \bar{b})^{T} e^{-i \omega_{0} \tau_{j} \theta} \bar{z}+\cdots .
\end{aligned}
$$

By virtue of (72), (91), and (92), it derives that

$$
\begin{aligned}
g(z, \bar{z}) & =\bar{q}^{*}(0) f_{0}(z, \bar{z}) \\
& =\bar{q}^{*}(0) f\left(0, y_{t}\right) \\
& =\tau_{j} \bar{J}\left(\begin{array}{c}
-\frac{r}{k} y_{1 t}^{2}(0)-\beta y_{1 t}(0) y_{2 t}(-1) \\
\beta y_{1 t}(0) y_{2 t}(-1) \\
\sum_{j=1}^{\infty} f_{j}^{(3)} y_{3 t}^{j}(0)
\end{array}\right) \\
& =\tau_{j} \bar{J}\left[\begin{array}{c}
r \\
-\frac{r}{k} y_{1 t}^{2}(0)-\beta y_{1 t}(0) y_{2 t}(-1)
\end{array}\right.
\end{aligned}
$$

$$
\begin{gathered}
\left.+\bar{a}^{*} \beta y_{1 t}(0) y_{2 t}(-1)+\sum_{j=1}^{\infty} \bar{b}^{*} f_{j}^{(3)} y_{3 t}^{j}(0)\right] \\
=\tau_{j} \bar{J}\left[-\frac{r}{k} y_{1 t}^{2}(0)+\left(\bar{a}^{*}-1\right) \beta y_{1 t}(0) y_{2 t}(-1)\right. \\
\left.+\sum_{j=1}^{\infty} \bar{b}^{*} f_{j}^{(3)} y_{3 t}^{j}(0)\right] \\
=-\frac{r}{k} \tau_{j} \bar{J}\left[z+\bar{z}+W_{20}^{(1)}(0) \frac{z^{2}}{2}+W_{11}^{(1)}(0) z \bar{z}\right. \\
\left.+W_{02}^{(1)}(0) \frac{\bar{z}^{2}}{2}+o\left(|(z, \bar{z})|^{3}\right)\right]^{2} \\
+\left(\bar{a}^{*}-1\right) \beta \tau_{j} \bar{J}
\end{gathered}
$$$$
\times\left[z+\bar{z}+W_{20}^{(1)}(0) \frac{z^{2}}{2}+W_{11}^{(1)}(0) z \bar{z}\right.
$$

$$
\begin{gathered}
\left.+W_{02}^{(1)}(0) \frac{\bar{z}^{2}}{2}+o\left(|z, \bar{z}|^{3}\right)\right] \\
\times\left[e^{-i \omega_{0} \tau_{j}} z+e^{i \omega_{0} \tau_{j}} \bar{z}+W_{20}^{(2)}(-1) \frac{z^{2}}{2}\right.
\end{gathered}
$$

$$
\begin{array}{r}
\left.+W_{11}^{(2)}(-1) z \bar{z}+W_{02}^{(2)}(-1) \frac{\bar{z}^{2}}{2}+o\left(|z, \bar{z}|^{3}\right)\right] \\
+\tau_{j} \bar{j}^{*}\left[f _ { 1 } ^ { ( 3 ) } \left(z+\bar{z}+W_{20}^{(3)}(0) \frac{z^{2}}{2}+W_{11}^{(3)}(0) z \bar{z}\right.\right. \\
\left.+W_{02}^{(1)}(0) \frac{\bar{z}^{2}}{2}+o\left(|z, \bar{z}|^{3}\right)\right) \\
+f_{2}^{(3)}\left(z+\bar{z}+W_{20}^{(3)}(0) \frac{z^{2}}{2}\right. \\
+W_{11}^{(3)}(0) z \bar{z}+W_{02}^{(1)}(0) \frac{\bar{z}^{2}}{2} \\
\left.\left.+o\left(|z, \bar{z}|^{3}\right)\right)+\cdots\right] .
\end{array}
$$


By comparing the coefficients with (91), it gives that

$$
\begin{aligned}
g_{20}=2 \tau_{j} \bar{J}[ & -\frac{r}{k}+\left(\bar{a}^{*}-1\right) \beta e^{-i \omega_{0} \tau_{j}} \\
& \left.+\bar{b}^{*}\left(\frac{f_{1}^{(3)} W_{20}^{(3)}(0)}{2}+f_{2}^{(3)}\right)\right], \\
g_{11}=\tau_{j} \bar{J}[ & -\frac{2 r}{k}+2\left(\bar{a}^{*}-1\right) \beta \cos \omega_{0} \tau_{j} \\
& \left.+f_{1}^{(3)} W_{11}^{(3)}(0)+2 f_{2}^{(3)}\right], \\
g_{02}=2 \tau_{j} \bar{J} & -\frac{r}{k}+\left(\bar{a}^{*}-1\right) \beta e^{-i \omega_{0} \tau_{j}} \\
& \left.+\bar{b}^{*}\left(\frac{f_{1}^{(3)} W_{02}^{(3)}(0)}{2}+f_{2}^{(3)}\right)\right], \\
& {\left[\beta\left(\bar{a}^{*}-1\right)\right.} \\
& \left.+f_{2}^{(3)}\left(W_{20}^{(3)}(0)+2 W_{11}^{(3)}(0)\right)\right] . \\
& +\left(W_{11}^{(2)}(-1)+\frac{W_{20}^{(3)}(-1)+W_{20}^{(1)}(0) e^{i \omega_{0} \tau_{j}}}{2}\right. \\
& \left.+W_{11}^{(1)}(0) e^{-i \omega_{0} \tau_{j}}\right) \\
g_{21}(1) & \left.(0)+2 W_{11}^{(1)}(0)\right)
\end{aligned}
$$

Since $g_{21}$ is associated with $W_{20}(\theta)$ and $W_{11}(\theta)$, further attempts should be carried out to compute $W_{20}(\theta)$ and $W_{11}(\theta)$.

By virtue of (78) and (86), we have

$$
\begin{aligned}
\dot{W} & =\dot{y}_{t}-\dot{z} q-\dot{\bar{z}} \bar{q} \\
& = \begin{cases}A W-2 \operatorname{Re}\left\{\bar{q}^{*}(0) f_{0} q(\theta)\right\}, & \theta \in[-1,0) \\
A W-2 \operatorname{Re}\left\{\bar{q}^{*}(0) f_{0} q(0)+f_{0}\right\}, & \theta=0 .\end{cases} \\
& \triangleq A W+H(z, \bar{z}, \theta),
\end{aligned}
$$

where

$$
H(z, \bar{z}, \theta)=H_{20}(\theta) \frac{z^{2}}{2}+H_{11}(\theta) z \bar{z}+H_{02}(\theta) \frac{\bar{z}^{2}}{2}+\cdots .
$$

By substituting the corresponding series into (95) and comparing the coefficients, we have

$$
\begin{gathered}
\left(A-2 i \omega_{0} \tau_{j}\right) W_{20}(\theta)=-H_{20}(\theta), \\
A W_{11}(\theta)=-H_{11}(\theta), \ldots .
\end{gathered}
$$

It follows from (95) that for $\theta \in[-1,0)$

$$
\begin{aligned}
H(z, \bar{z}, \theta) & =-\bar{q}^{*}(0) f_{0} q(\theta)-q^{*}(0) \bar{f}_{0} \bar{q}(\theta) \\
& =-g(z, \bar{z}) q(\theta)-\bar{g}(z, \bar{z}) \bar{q}(\theta) .
\end{aligned}
$$

By comparing coefficients in (96) with those in (94), it derives that

$$
\begin{aligned}
& H_{20}(\theta)=-g_{20} q(\theta)-\bar{g}_{02} \bar{q}(\theta), \\
& H_{11}(\theta)=-g_{11} q(\theta)-\bar{g}_{11} \bar{q}(\theta) .
\end{aligned}
$$

Based on the definition of $A$ and (97) and (99), it can be obtained that

$$
\begin{aligned}
& \dot{W}_{20}(\theta)=2 i \omega_{0} \tau_{j} W_{20}(\theta)+g_{20} q(\theta)+\bar{g}_{02} \bar{q}(\theta) \\
& \text { For } q(\theta)=(1, a, b)^{T} e^{i \omega_{0} \tau_{j} \theta}, \\
& W_{20}(\theta)=\frac{i g_{20}}{\omega_{0} \tau_{j}} q(0) e^{i \omega_{0} \tau_{j} \theta}+\frac{i \bar{g}_{02}}{3 \omega_{0} \tau_{j}} \bar{q}(0) e^{-i \omega_{0} \tau_{j} \theta}+G_{1} e^{2 i \omega_{0} \tau_{j} \theta},
\end{aligned}
$$

where $G_{1}=\left(G_{1}^{(1)}, G_{1}^{(2)}, G_{1}^{(3)}\right)$ is a constant vector.

Similarly, it follows from (97) and (100) that

$$
W_{11}(\theta)=-\frac{i g_{11}}{\omega_{0} \tau_{j}} q(0) e^{i \omega_{0} \tau_{j} \theta}+\frac{i \bar{g}_{11}}{\omega_{0} \tau_{j}} \bar{q}(0) e^{-i \omega_{0} \tau_{j} \theta}+G_{2},
$$

where $G_{2}=\left(G_{2}^{(1)}, G_{2}^{(2)}, G_{2}^{(3)}\right)$ is a constant vector.

Subsequently, values of $G_{1}$ and $G_{2}$ should be computed. By using the definition of $A$ and (95), we have

$$
\begin{gathered}
\int_{-1}^{0} \mathrm{~d} \eta w_{20}(\theta)=2 i \omega_{0} \tau_{j} W_{20}(0)-H_{20}(0), \\
\int_{-1}^{0} \mathrm{~d} \eta(\theta) W_{11}(\theta)=-H_{11}(0),
\end{gathered}
$$

where $\eta(\theta)=\eta(0, \theta)$. Based on (95), it derives that in the case of $\theta=0$,

$$
\begin{aligned}
H(z, \bar{z}, 0) & =-2 \operatorname{Re}\left\{\bar{q}^{*}(0) f_{0} q(0)\right\}+f(0) \\
& =-\bar{q}^{*}(0) f_{0} q(0)-q^{*}(0) \bar{f}_{0} \bar{q}(0)+f_{0} \\
& =-g(z, \bar{z}) q(0)-\bar{g}(z, \bar{z}) \bar{q}(0)+f_{0},
\end{aligned}
$$

which follows that

$$
\begin{aligned}
H_{20}( & (\theta) \frac{z^{2}}{2}+H_{11}(\theta) z \bar{z}+H_{02}(\theta) \frac{\bar{z}^{2}}{2}+\cdots \\
= & -q(0)\left(g_{20} \frac{z^{2}}{2}+g_{11} z \bar{z}+g_{02} \frac{\bar{z}^{2}}{2}+\cdots\right) \\
& -\bar{q}(0)\left(\bar{g}_{20} \frac{\bar{z}^{2}}{2}+\bar{g}_{11} z \bar{z}+\bar{g}_{02} \frac{z^{2}}{2}+\cdots\right)+f_{0} .
\end{aligned}
$$


By virtue of (72), it gives that

$$
f_{0}=\tau_{k}\left(\begin{array}{c}
-\frac{r}{k} y_{1 t}^{2}(0)-\beta y_{1 t}(0) y_{2 t}(-1) \\
\beta y_{1 t}(0) y_{2 t}(-1) \\
\sum_{j=1}^{\infty} f_{j}^{(3)} y_{3 t}^{j}(0)
\end{array}\right)
$$

By virtue of (86), it can be obtained that

$$
\begin{aligned}
y_{t}(\theta)= & W(t, \theta)+2 \operatorname{Re}\{z(t) q(\theta)\} \\
= & W(t, \theta)+z(t) q(\theta)+\bar{z}(t) \bar{q}(\theta) \\
= & W_{20}(\theta) \frac{z^{2}}{2}+W_{21}(\theta) z \bar{z}+z(t) q(\theta) \\
& +\bar{z}(t) \bar{q}(\theta)+\cdots .
\end{aligned}
$$

Then we have

$$
\begin{aligned}
& f_{0}=\tau_{j}\left(\begin{array}{c}
-\frac{r}{k}-\beta e^{-i \omega_{0} \tau_{j}} \\
\beta e^{-i \omega_{0} \tau_{j}} \\
f_{1}^{(3)} \frac{W_{20}^{(3)}(0)}{2}+f_{2}^{(3)}
\end{array}\right) z^{2} \\
& +\tau_{k}\left(\begin{array}{c}
-\frac{2 r}{k}-2 \beta \cos \omega_{0} \tau_{j} \\
2 \beta \cos \omega_{0} \tau_{j} \\
2 f_{2}^{(3)}+f_{1}^{(3)} W_{11}^{(3)}(0)
\end{array}\right) z \bar{z}+\cdots
\end{aligned}
$$

According to (107) and (110), we have

$$
\begin{aligned}
H_{20}(0)= & -g_{20} q(0)-\bar{g}_{02} \bar{q}(0) \\
& +2 \tau_{j}\left(\begin{array}{c}
-\frac{r}{k}-\beta e^{-i \omega_{0} \tau_{j}} \\
\beta e^{-i \omega_{0} \tau_{j}} \\
f_{1}^{(3)} \frac{W_{20}^{(3)}(0)}{2}+f_{2}^{(3)}
\end{array}\right), \\
H_{11}(0)= & -g_{11} q(0)-\bar{g}_{11} \bar{q}(0) \\
& +\tau_{j}\left(\begin{array}{c}
-\frac{2 r}{k}-2 \beta \cos \omega_{0} \tau_{j} \\
2 \beta \cos \omega_{0} \tau_{j} \\
2 f_{2}^{(3)}+f_{1}^{(3)} W_{11}^{(3)}(0)
\end{array}\right) .
\end{aligned}
$$

Since $i \omega_{0} \tau_{j}$ is the eigenvalue of $A(0)$ and $q(0)$ is the corresponding eigenvector, we obtain that

$$
\begin{gathered}
\left(i \omega_{0} \tau_{j} I-\int_{-1}^{0} e^{i \omega_{0} \tau_{j} \theta} \mathrm{d} \eta(\theta)\right) q(0)=0, \\
\left(-i \omega_{0} \tau_{j} I-\int_{-1}^{0} e^{-i \omega_{0} \tau_{j} \theta} \mathrm{d} \eta(\theta)\right) \bar{q}(0)=0,
\end{gathered}
$$

where $I$ is identity matrix.

By substituting (102) and (111) into (104), it can be obtained that

$$
\begin{array}{r}
\left(2 i \omega_{0} \tau_{j} I-\int_{-1}^{0} e^{2 i \omega_{0} \tau_{j} \theta} \mathrm{d} \eta(\theta)\right) G_{1} \\
\quad=2 \tau_{j}\left(\begin{array}{c}
-\frac{r}{k}-\beta e^{-i \omega_{0} \tau_{j}} \\
\beta e^{-i \omega_{0} \tau_{j}} \\
f_{1}^{(3)} \frac{W_{20}^{(3)}(0)}{2}+f_{2}^{(3)}
\end{array}\right),
\end{array}
$$

which can be rewritten as follows:

$$
\left(\begin{array}{ccc}
2 i \omega_{0}+\frac{r \widetilde{S}^{*}}{k} & \beta \widetilde{S}^{*} e^{-2 i \omega_{0} \tau_{j}} & 0 \\
-\beta \widetilde{I}^{*} & 2 i \omega_{0}+\left(\mu_{1}+m\right)-\beta \widetilde{S}^{*} e^{-2 i \omega_{0} \tau_{j}} & 0 \\
0 & -m & 2 i \omega_{0}+\mu_{2}-\frac{c v^{*}}{\left(w \widetilde{R}^{*}-c\right)^{2}}
\end{array}\right) G_{1}=2\left(\begin{array}{c}
-\frac{r}{k}-\beta e^{-i \omega_{0} \tau_{j}} \\
\beta e^{-i \omega_{0} \tau_{j}} \\
\frac{W_{20}^{(3)}(0)}{2}+f_{2}^{(3)}
\end{array}\right)
$$

Based on Grammar Law [43], $G_{1}^{(1)}, G_{1}^{(2)}$, and $G_{1}^{(3)}$ can be obtained as follows:

$$
G_{1}^{(1)}=\frac{2}{U_{1}}\left|\begin{array}{ccc}
-\frac{r}{k}-\beta e^{-i \omega_{0} \tau_{j}} & \beta \widetilde{S}^{*} e^{-2 i \omega_{0} \tau_{j}} & 0 \\
\beta e^{-i \omega_{0} \tau_{j}} & 2 i \omega_{0}+\left(\mu_{1}+m\right)-\beta \widetilde{S}^{*} e^{-2 i \omega_{0} \tau_{j}} & 0 \\
f_{1}^{(3)} \frac{W_{20}^{(3)}(0)}{2}+f_{2}^{(3)} & -m & 2 i \omega_{0}+\mu_{2}-\frac{c v^{*}}{\left(w \widetilde{R}^{*}-c\right)^{2}}
\end{array}\right|,
$$




$$
\begin{aligned}
& G_{1}^{(2)}=\frac{2}{U_{1}}\left|\begin{array}{ccc}
2 i \omega_{0}+\frac{r \widetilde{S}^{*}}{k} & -\frac{r}{k}-\beta e^{-i \omega_{0} \tau_{j}} & 0 \\
-\beta \widetilde{I}^{*} & \beta e^{-i \omega_{0} \tau_{j}} & 0 \\
0 & f_{1}^{(3)} \frac{W_{20}^{(3)}(0)}{2}+f_{2}^{(3)} & 2 i \omega_{0}+\mu_{2}-\frac{c v^{*}}{\left(w \widetilde{R}^{*}-c\right)^{2}}
\end{array}\right|, \\
& G_{1}^{(3)}=\frac{2}{U_{1}}\left|\begin{array}{ccc}
2 i \omega_{0}+\frac{r \widetilde{S}^{*}}{k} & \beta \widetilde{S}^{*} e^{-2 i \omega_{0} \tau_{j}} & -\frac{r}{k}-\beta e^{-i \omega_{0} \tau_{j}} \\
-\beta \widetilde{I}^{*} & 2 i \omega_{0}+\left(\mu_{1}+m\right)-\beta \widetilde{S}^{*} e^{-2 i \omega_{0} \tau_{j}} & \beta e^{-i \omega_{0} \tau_{j}} \\
0 & -m & f_{1}^{(3)} \frac{W_{20}^{(3)}(0)}{2}+f_{2}^{(3)}
\end{array}\right|,
\end{aligned}
$$

where

$$
U_{1}=\left|\begin{array}{ccc}
2 i \omega_{0}+\frac{r \widetilde{S}^{*}}{k} & \beta \widetilde{S}^{*} e^{-2 i \omega_{0} \tau_{j}} & 0 \\
-\beta \widetilde{I}^{*} & 2 i \omega_{0}+\left(\mu_{1}+m\right)-\beta \widetilde{S}^{*} e^{-2 i \omega_{0} \tau_{j}} & 0 \\
0 & -m & 2 i \omega_{0}+\mu_{2}-\frac{c v^{*}}{\left(w \widetilde{R}^{*}-c\right)^{2}}
\end{array}\right| .
$$

Similarly, substituting (103) and (112) into (105), it can be obtained that

$$
\begin{aligned}
& \left(\begin{array}{ccc}
\frac{r \widetilde{S}^{*}}{k} & \beta \widetilde{S}^{*} & 0 \\
-\beta \widetilde{I}^{*} & \mu_{1}+m-\beta \widetilde{S}^{*} & 0 \\
0 & -m & \mu_{2}-\frac{c v^{*}}{\left(w \widetilde{R}^{*}-c\right)^{2}}
\end{array}\right) G_{2} \\
& =2\left(\begin{array}{c}
-\frac{2 r}{k}-2 \beta \cos \omega_{0} \tau_{j} \\
2 \beta \cos \omega_{0} \tau_{j} \\
2 f_{2}^{(3)}+f_{1}^{(3)} W_{11}^{(3)}(0)
\end{array}\right)
\end{aligned}
$$

Based on Grammar Law [43], $G_{2}^{(1)}, G_{2}^{(2)}$, and $G_{2}^{(3)}$ can be obtained as follows:

$$
\begin{aligned}
& G_{2}^{(1)} \\
& =\frac{1}{U_{2}}\left|\begin{array}{ccc}
-\frac{2 r}{k}-2 \beta \cos \omega_{0} \tau_{j} & \beta \widetilde{S}^{*} & 0 \\
2 \beta \cos \omega_{0} \tau_{j} & \mu_{1}+m-\beta \widetilde{S}^{*} & 0 \\
2 f_{2}^{(3)}+f_{1}^{(3)} W_{11}^{(3)}(0) & -m & \mu_{2}-\frac{c v^{*}}{\left(w \widetilde{R}^{*}-c\right)^{2}}
\end{array}\right|, \\
& G_{2}^{(2)} \\
& =\frac{1}{U_{2}}\left|\begin{array}{ccc}
\frac{r \widetilde{S}^{*}}{k} & -\frac{2 r}{k}-2 \beta \cos \omega_{0} \tau_{j} & 0 \\
-\beta \widetilde{I}^{*} & 2 \beta \cos \omega_{0} \tau_{j} & 0 \\
0 & 2 f_{2}^{(3)}+f_{1}^{(3)} W_{11}^{(3)}(0) & \mu_{2}-\frac{c v^{*}}{\left(w \widetilde{R}^{*}-c\right)^{2}}
\end{array}\right|,
\end{aligned}
$$

$$
\begin{aligned}
& G_{2}^{(3)} \\
& =\frac{1}{U_{2}}\left|\begin{array}{ccc}
\frac{r \widetilde{S}^{*}}{k} & \beta \widetilde{S}^{*} & -\frac{2 r}{k}-2 \beta \cos \omega_{0} \tau_{j} \\
-\beta \widetilde{I}^{*} & \mu_{1}+m-\beta \widetilde{S}^{*} & 2 \beta \cos \omega_{0} \tau_{j} \\
0 & -m & 2 f_{2}^{(3)}+f_{1}^{(3)} W_{11}^{(3)}(0)
\end{array}\right|,
\end{aligned}
$$

where

$$
U_{2}=\left|\begin{array}{ccc}
\frac{r \widetilde{S}^{*}}{k} & \beta \widetilde{S}^{*} & 0 \\
-\beta \widetilde{I}^{*} & \mu_{1}+m-\beta \widetilde{S}^{*} & 0 \\
0 & -m & \mu_{2}-\frac{c v^{*}}{\left(w \widetilde{R}^{*}-c\right)^{2}}
\end{array}\right| .
$$

It follows from the above computation and analysis that $W_{20}(\theta)$ and $W_{11}(\theta)$ can be determined based on (102) and (103).

Furthermore, we can compute $g_{21}$ based on (94). Hence, the following values can be computed as follows:

$$
\begin{aligned}
d_{1}(0) & =\frac{i}{2 \omega_{0} \tau_{j}}\left(g_{20} g_{11}-2\left|g_{11}\right|^{2}-\frac{\left|g_{02}\right|^{2}}{3}\right)+\frac{g_{21}}{2}, \\
\delta_{2} & =-\frac{\operatorname{Re}\left\{d_{1}(0)\right\}}{\operatorname{Re}\left\{\lambda^{\prime}\left(\tau_{j}\right)\right\}}, \\
\gamma_{2} & =2 \operatorname{Re}\left(d_{1}(0)\right), \\
T_{2} & =\frac{\operatorname{Im}\left\{d_{1}(0)\right\}+\delta_{2} \operatorname{Im}\left\{\lambda^{\prime}\left(\tau_{j}\right)\right\}}{\omega_{0} \tau_{j}} .
\end{aligned}
$$


TABLE 2: Values of parameters for numerical simulation.

\begin{tabular}{lc}
\hline Parameter & Value \\
\hline$r$ & 0.1 \\
$k$ & 1 \\
$\beta$ & 1 \\
$m$ & 0.1 \\
$\mu_{1}$ & 0.1 \\
$\mu_{2}$ & 0.1 \\
$w$ & 20 \\
$c$ & 1 \\
\hline
\end{tabular}

By using similar arguments in [49], some properties of bifurcating periodic solutions of model (4) in the center manifold at the critical values are discussed in this paper. Based on the analysis in Section 4.2.2 of this paper, the following theorem can be concluded.

Theorem 19. The properties of Hopf bifurcation are determined by values in (121).

(i) $\delta_{2}$ determines directions of Hopf bifurcation: if $\delta_{2}>$ $0\left(\delta_{2}<0\right)$, then Hopf bifurcation is supercritical (subcritical) and the bifurcating periodic solutions exist for $\tau>\tau_{j}\left(\tau<\tau_{j}\right)$.

(ii) $\gamma_{2}$ determines the stability of bifurcating periodic solutions: bifurcating periodic solutions are stable (unstable) if $\gamma_{2}<0\left(\gamma_{2}>0\right)$.

(iii) $T_{2}$ determines the period of bifurcating periodic solutions: period increases (decreases) if $T_{2}>0\left(T_{2}<0\right)$.

\section{Numerical Simulation}

In this section, some numerical simulations are provided to substantiate the theoretical results obtained in Section 4 of this paper.

5.1. Numerical Simulation of State Feedback Controller for Singularity Induced Bifurcation and Local Stability Switch. In this subsection, values of parameters are partially taken from Section 5 of [11] and set in appropriate units, which can be found in Table 2. Numerical simulations are provided to illustrate the effectiveness of the state feedback controllers designed in Section 4.1 in the case of zero economic interest and positive economic interest, respectively.

In the case of zero economic interest, model (4) without time delay takes the following form:

$$
\begin{aligned}
\dot{S}(t) & =0.1(1-S(t))-S(t) I(t), \\
\dot{I}(t) & =S(t) I(t)-0.1 I(t)-0.1 I(t), \\
\dot{R}(t) & =0.1 I(t)-0.1 R(t)-E(t) R(t), \\
0 & =E(t)(20 R(t)-1) .
\end{aligned}
$$

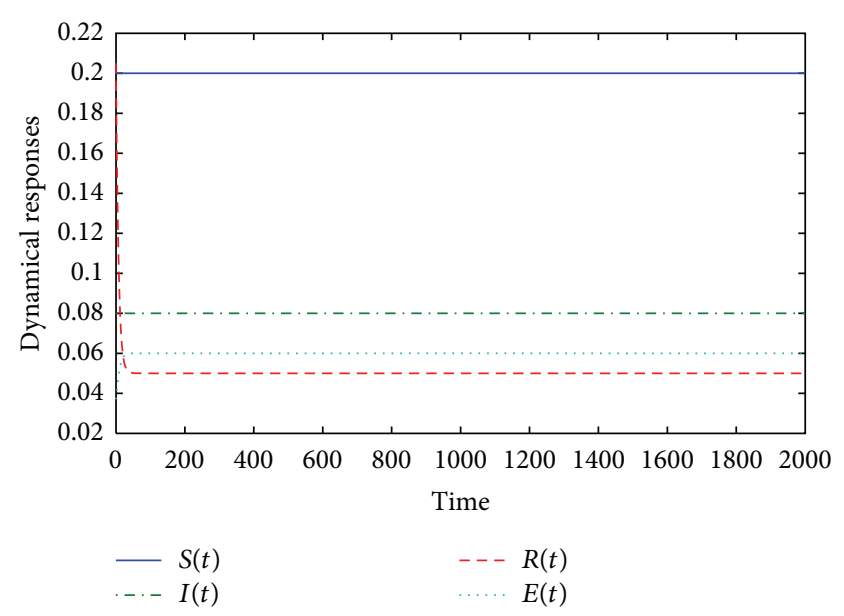

FIGURE 1: Dynamical responses of model system (123) with state feedback controller, which shows that model system (123) is stable around $(0.2,0.08,0.05,0.06)$.

By using Theorem 11 of this paper, it can be shown that the model (4) without time delay has a singularity induced bifurcation around the interior equilibrium $P^{*}(0.2,0.08,0.05,0.06)$, and local stability switch occurs as $v$ increases through 0 .

Based on the analysis in Section 4.1.2 and Theorem 14 of this paper, a state feedback controller $u(t)=l(E(t)-0.06)$ can be applied to stabilize model system (122) around $P^{*}$, and then the model system (122) with the state feedback controller takes the following form:

$$
\begin{aligned}
\dot{S}(t) & =0.1(1-S(t))-S(t) I(t), \\
\dot{I}(t) & =S(t) I(t)-0.1 I(t)-0.1 I(t), \\
\dot{R}(t) & =0.1 I(t)-0.1 R(t)-E(t) R(t), \\
0 & =E(t)(20 R(t)-1)+l(E(t)-0.06) .
\end{aligned}
$$

By using Theorem 14 of this paper, if the feedback gain $l$ satisfies $l>2.2429$, then model system (122) is stable around $P^{*}$ and singularity induced bifurcation of the model system (122) is also eliminated. The dynamical responses of model system (122) can be shown in Figure 1.

Furthermore, based on the analysis and inequality (55) in Section 4.1.2, there are two interior equilibria (denoted by $\widetilde{P}_{1}^{*}$ and $\widetilde{P}_{2}^{*}$ ) when $0<v<0.00668$. In the following part, we focus on the case of $0<v<0.00668$, and the economic interest is set as $v^{*}=0.005 \in(0,0.00668)$ in appropriate unit, which is arbitrarily selected within the interval $(0,0.00668)$ and is enough to merit the theoretical analysis obtained in Section 4.1.2. By virtue of the given values of parameters in Table 2 and (54), two interior equilibria can be obtained as follows: $\widetilde{P}_{1}^{*}(0.2,0.08,0.0557,0.0439)$ and $\widetilde{P}_{2}^{*}(0.2,0.08,0.07175,0.0115)$. By using Theorem 11 of this 
paper, it can be shown that model system (124) is unstable around $\widetilde{P}_{1}^{*}$ and $\widetilde{P}_{2}^{*}$ :

$$
\begin{aligned}
\dot{S}(t) & =0.1(1-S(t))-S(t) I(t), \\
\dot{I}(t) & =S(t) I(t)-0.1 I(t)-0.1 I(t), \\
\dot{R}(t) & =0.1 I(t)-0.1 R(t)-E(t) R(t), \\
0 & =E(t)(20 R(t)-1)-0.005 .
\end{aligned}
$$

Based on the analysis in Section 4.1.2 of this paper, state feedback controllers $u(t)=\tilde{l}(E(t)-0.0439)$ and $u(t)=$ $\widetilde{l}(E(t)-0.0115)$ can be applied to stabilize the model system (124) around $\widetilde{P}_{1}^{*}$ and $\widetilde{P}_{2}^{*}$, respectively. The model system (124) with respective state feedback controller takes the following form:

$$
\begin{aligned}
\dot{S}(t) & =0.1(1-S(t))-S(t) I(t), \\
\dot{I}(t) & =S(t) I(t)-0.1 I(t)-0.1 I(t), \\
\dot{R}(t) & =0.1 I(t)-0.1 R(t)-E(t) R(t), \\
0 & =E(t)(20 R(t)-1)-0.005+\tilde{l}(E(t)-0.0439), \\
\dot{S}(t) & =0.1(1-S(t))-S(t) I(t), \\
\dot{I}(t) & =S(t) I(t)-0.1 I(t)-0.1 I(t), \\
\dot{R}(t) & =0.1 I(t)-0.1 R(t)-E(t) R(t), \\
0 & =E(t)(20 R(t)-1)-0.005+\widetilde{l}(E(t)-0.0115) .
\end{aligned}
$$

By using Theorem 15 of this paper, if the feedback gain $\tilde{l}$ satisfies $\widetilde{l}>3.753$, then model system (125) is stable around $\widetilde{P}_{1}^{*}$ and model system $(126)$ is stable around $\widetilde{P}_{2}^{*}$. The dynamical responses of model system (125) and (126) can be shown in Figures 2 and 3, respectively.

5.2. Numerical Simulation for Hopf Bifurcation and Local Stability Switch. In this subsection,values of parameters are partially taken from Section 5 of [11] and set in appropriate units, which can be found in Table 3. Numerical simulations are provided to support the theoretical findings obtained in Section 4.2 of this paper.

Based on the analysis and inequality (55) in Section 4.1.2, there are two interior equilibria (denoted by $\widetilde{P}_{1}^{*}$ and $\widetilde{P}_{2}^{*}$ ) when $0<v<0.01831$. In the following part, we focus on the case of $0<v<0.01831$, and the economic interest is set as $v^{*}=0.012 \in(0,0.01831)$ in appropriate unit, which is arbitrarily selected within the interval $(0,0.01831)$ and is enough to merit the theoretical analysis obtained in Section 4.2. By virtue of the given values of parameters in Table 3 and (54), two interior equilibria can be obtained as follows: $\widetilde{P}_{1}^{*}(0.2,0.08,0.0907,0.0147)$ and $\widetilde{P}_{2}^{*}(0.2,0.08,0.0573,0.0821)$. Furthermore, it can be computed that $k \beta>3\left(\mu_{1}+m\right)$. Based on the analysis in

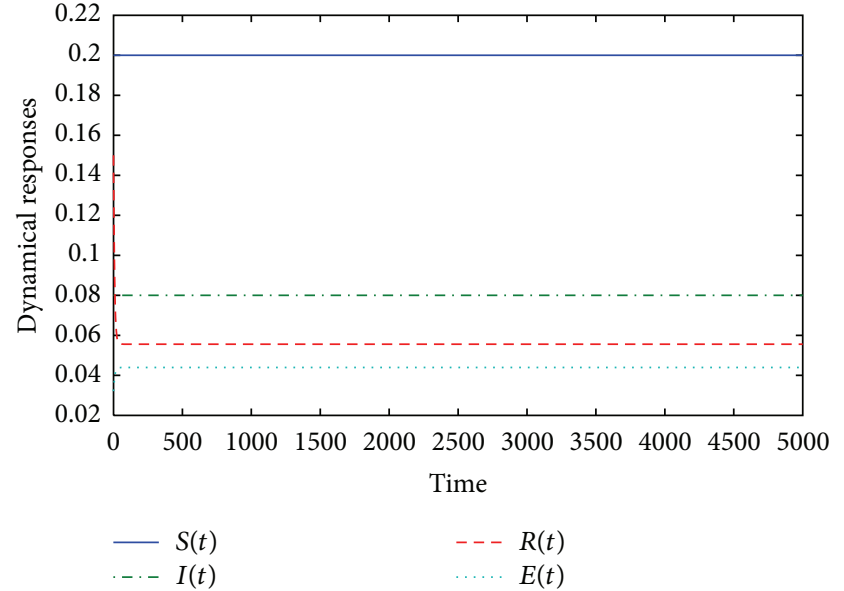

FIGURE 2: Dynamical responses of model system (125) with state feedback controller, which shows that model system (125) is stable around $(0.2,0.08,0.0557,0.0439)$.

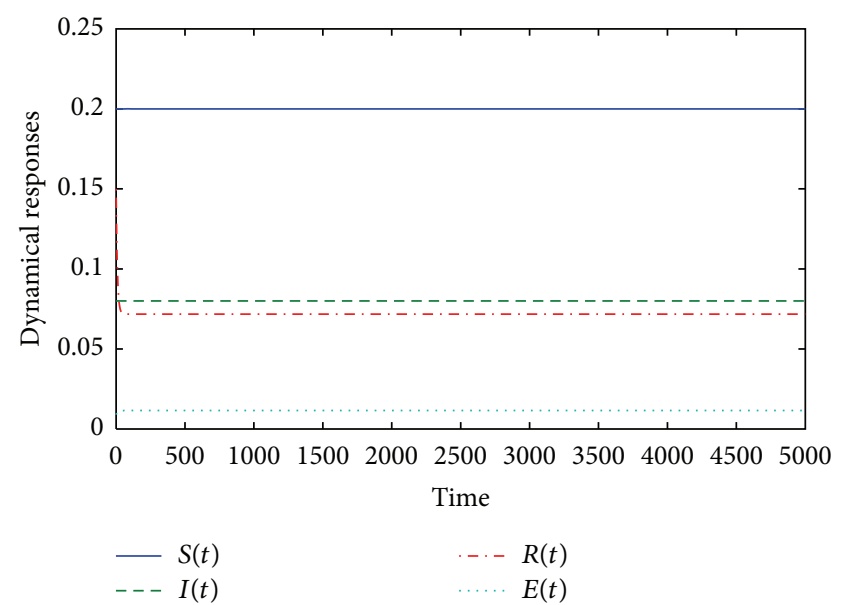

Figure 3: Dynamical responses of model system (126) with state feedback controller, which shows that model system (126) is stable around $(0.2,0.08,0.07175,0.0115)$.

TABLE 3: Values of parameters for numerical simulation.

\begin{tabular}{lc}
\hline Parameter & Value \\
\hline$r$ & 0.1 \\
$k$ & 1 \\
$\beta$ & 1 \\
$m$ & 0.13 \\
$\mu_{1}$ & 0.1 \\
$\mu_{2}$ & 0.1 \\
$w$ & 20 \\
$c$ & 1 \\
\hline
\end{tabular}

Section 4.2.1, it satisfies the assumption that (58) has a positive root, and then the corresponding $\tau_{0}^{*}=2.7814$ can be calculated by solving (64). It follows from (121) that $\delta_{2}=1.2963>0, \gamma_{2}=-0.3012<0$, and $T_{2}=$ $1.2467>0$. Consequently, the interior equilibrium $\widetilde{P}_{1}^{*}$ 

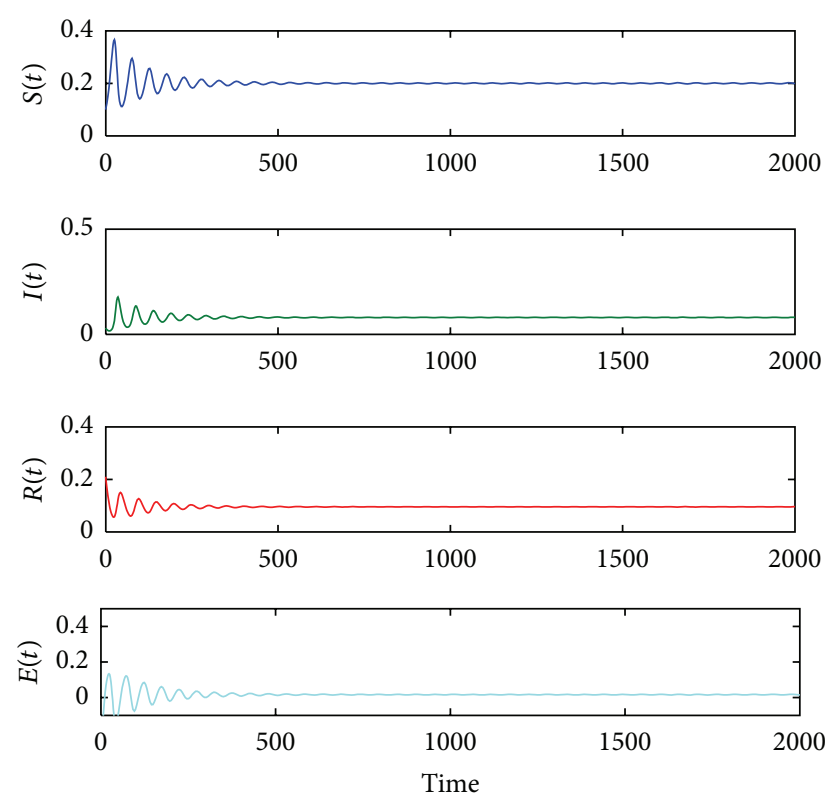

FIGURE 4: Dynamical responses of model system (4) with time delay $\tau=1.54$, which shows that model system (4) is stable around $(0.2,0.08,0.0907,0.0147)$.
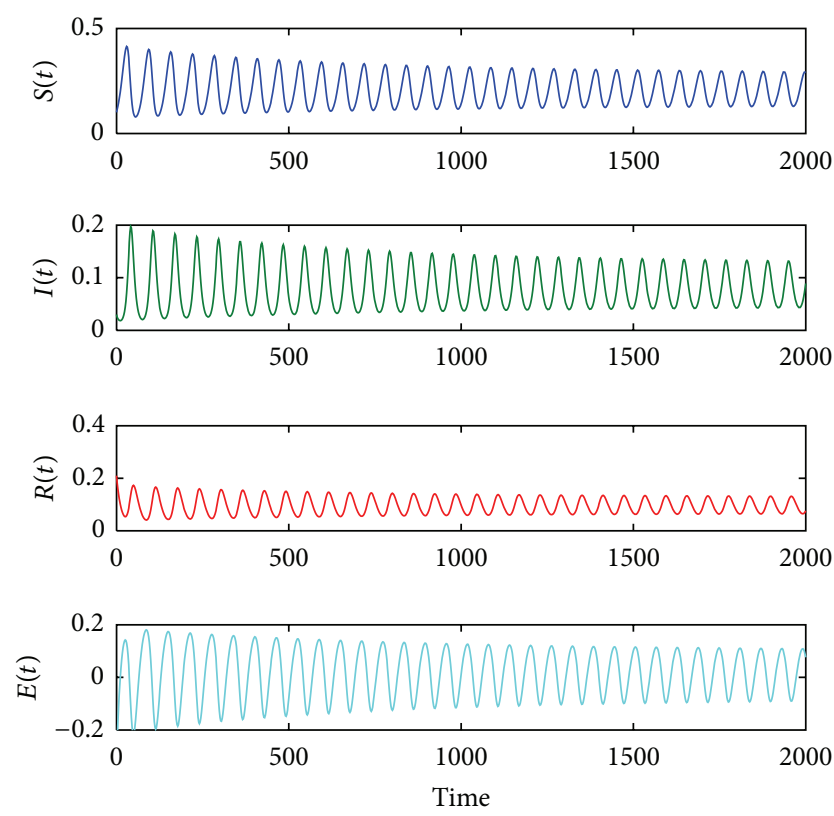

FIGURE 5: Dynamical responses of model system (4) with time delay $\tau=3$, which shows that model system (4) is unstable around $(0.2,0.08,0.0907,0.0147)$.

remains stable for $\tau<\tau_{0}^{*}$, and dynamical responses of model system (4) with $\tau=1.54$ are plotted in Figure 4 . It should be noted that $\tau=1.54$ in Figure 4 is ran-domly selected in the interval $(0,2.7814)$, which is enough to merit the above mathematical study. Only the dynamical responses and corresponding phase portrait of model (4) around $\widetilde{P}_{1}^{*}$

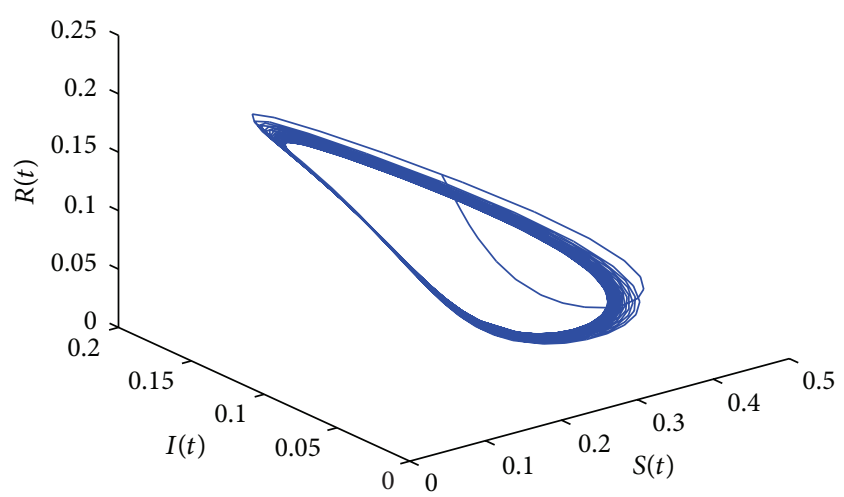

FIGURE 6: A limit cycle corresponding to the periodic solution in Figure 5 in the $S-I-R$ space.

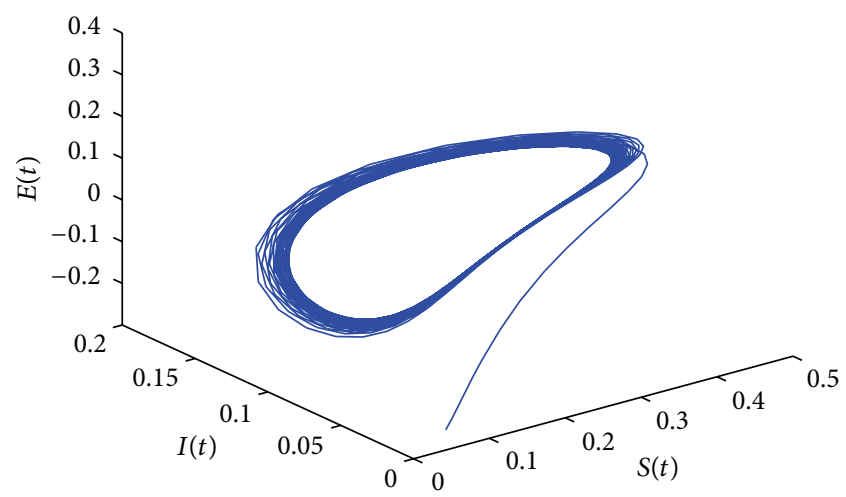

FIGURE 7: Limit cycle corresponding to the periodic solution in Figure 5 in the $S-I-E$ space.

are plotted; some symmetric results about $\widetilde{P}_{2}^{*}$ can be also obtained. As $\tau$ increases through $\tau_{0}^{*}$, a periodic solution caused by the phenomenon of Hopf bifurcation occurs; that is, a family of periodic solutions bifurcate from the interior equilibrium $\widetilde{P}_{1}^{*}$. Since $\delta_{2}>0$ and $\gamma_{2}<0$, the Hopf bifurcation is supercritical, the directions of the Hopf bifurcation is $\tau>$ $\tau_{0}^{*}$, and these bifurcating periodic solutions from the interior equilibrium $\widetilde{P}_{1}^{*}$ at $\tau_{0}^{*}$ are stable. Dynamical responses of model (4) with $\tau=3>\tau_{0}^{*}$ are plotted in Figure 5. Figures 6 and 7 show a limit cycle corresponding to the periodic solution in Figure 5 in the $S-I-R$ and $S-I-E$ space, respectively.

\section{Conclusion}

It is well known that the recovered host individuals are naturally immune to vector disease [1], and its potential economic interest can be commercially exploited. Furthermore, harvest effort is usually influenced by variation of economic interest under market economy. Consequently, it is necessary to discuss the coexistence and interaction mechanism of population within harvested epidemiological ecosystem as well as dynamical effect of harvest effort due to variation of economic interest. 
By introducing commercial harvest effort into model proposed in [11], a delayed hybrid mathematical model is established to investigate the dynamical effect of commercial harvesting and incubation time delay on epidemiological economic system, which extends the work done in [11] from a bioeconomic perspective. Positivity and persistence of solutions of model system are discussed in Theorems 3 and 10, respectively. The economic interest of commercial harvesting should be restricted within certain interval that guarantees the existence of interior equilibrium, which can be found in Remark 16. Since the interior equilibrium biologically interprets that susceptible, infective, and recovered host individuals survive as well as harvest on recovered host individuals exist, the bifurcation phenomena around the interior equilibria can reveal the instability mechanism of model system, which are theoretically relevant to infectious disease control and sustainable yield on recovered host individuals. Consequently, we will mainly concentrate on dynamical behavior and local stability switch around interior equilibrium of model system (4) in this paper. As analyzed in Theorem 11 of this paper, a singularity induced bifurcation occurs which leads to local stability switch in the case of positive economic interest of harvesting. In the perspective of practical viewpoint, a direct damage done by the singularity induced bifurcation to the proposed model is impulse phenomenon, which may lead to outbreak of infectious disease and hamper prosperous harvesting on recovered host individual population resource in the harvested ecosystem, which can be found in Remark 13. With the purpose of maintaining the economic interest at an ideal level, state feedback controllers are designed to stabilize model system around the desirable interior equilibria in the case of zero economic interest and positive economic interest, respectively. The design of the state feedback controller can be found in Theorems 14 and 15 of this paper. The theoretical results and numerical simulations obtained in this paper suggest that incorporating harvest effort on recovered host individuals can not only prevent the stability switch of model system,but also drive model system to stable equilibrium, which will contribute to the persistence and sustainable yield of the harvested ecosystem.

Further attempts are made to understand the dynamical effect of incubation time delay and economic interest on local stability of model system around interior equilibrium. Local stability analysis reveals that incubation delay is responsible for local stability switch of the proposed model, and a family of periodic solutions bifurcate from the interior equilibrium which occurs as incubation delay increases through a critical threshold, which can be found in Theorem 17. The direction and stability of Hopf bifurcation are also discussed in Theorem 19 of this paper, which reveals that Hopf bifurcation is supercritical, the directions of Hopf bifurcation is $\tau>\tau_{0}^{*}$, and these bifurcating periodic solutions from the interior equilibrium are stable. The model proposed in [11] does not discuss the harvest effort on economic population. For the model proposed in [11], the threshold value of incubation delay where Hopf bifurcation occurs in [11] is $\widehat{\tau}_{0}=2.0842$. However, the harvest effort on recovered host individuals is considered in this paper. As calculated in Section 5.2 of this paper, the Hopf bifurcation occurs at $\tau_{0}^{*}=2.7814$ in the case of positive economic interest. It is obvious that $\tau_{0}^{*}>\widehat{\tau}_{0}$, which implies that the harvesting has a stabilizing impact on the dynamical behavior of population dynamics; cyclic behavior caused by incubation delay can be deferred by introduction of commercial harvesting effort.

It should be noted that some hybrid dynamical models are proposed in [23-28], which are utilized to discuss the interaction mechanism of harvested ecosystem from an economic perspective in recent years. Complex dynamical behavior and stability analysis in prey-predator ecosystems with stagestructured population and gestation delay are considered. However, as far as knowledge goes, nobody has explicitly proposed a mathematical model to discuss the dynamic effect of commercial harvest on epidemiological system under the market economy environment. The main objective of this paper is to investigate the transmission mechanism of infectious disease and dynamical effect of commercial harvest on population dynamics, especially the complex dynamical behavior and stability switch due to variation of incubation and commercial harvest economic interest, which makes the work studied in this paper has some new and positive features.

\section{Conflict of Interests}

All authors of this paper declare that there is no conflict of interests regarding the publication of this paper. They have no proprietary, financial, professional, or other personal interest of any nature or kind in any product, service, and/or company that could be construed as influencing the position presented in, or the review of, this paper.

\section{Acknowledgments}

This work is supported by National Natural Science Foundation of China, Grant no. 61104003, Grant no. 61273008, and Grant no. 61104093; Research Foundation for Doctoral Program of Higher Education of Education Ministry, Grant no. 20110042120016; Hebei Province Natural Science Foundation, Grant no. F2011501023; Fundamental Research Funds for the Central Universities, Grant no. N120423009; and Research Foundation for Science and Technology Pillar Program of Northeastern University at Qinhuangdao, Grant no. XNK201301. This work is supported by State Key Laboratory of Integrated Automation of Process Industry, Northeastern University and supported by Hong Kong Admission Scheme for Mainland Talents and Professionals, Hong Kong Special Administrative Region.

\section{References}

[1] R. M. Anderson and R. M. May, "Population biology of infectious diseases: part I," Nature, vol. 280, no. 5721, pp. 361$367,1979$.

[2] S. A. Levin, T. G. Hallam, and J. J. Gross, Applied Mathematical Ecology, Springer, New York, NY, USA, 1990. 
[3] H. W. Hethcote, "The mathematics of infectious diseases," SIAM Review, vol. 42, no. 4, pp. 599-653, 2000.

[4] Z. E. Ma, Y. Zhou, W. Wang, and Z. Jin, Mathematical Models and Dynamics OfInfectious Disease, Science Press, Beijing, China, 2004.

[5] K. L. Cooke, "Stability analysis for a vector disease model," The Rocky Mountain Journal of Mathematics, vol. 9, no. 1, pp. 3142, 1979, Conference on Deterministic Differential Equations and Stochastic Processes Models for Biological Systems (San Cristobal, N.M., 1977).

[6] W. M. Liu, H. W. Hethcote, and S. A. Levin, "Dynamical behavior of epidemiological models with nonlinear incidence rates," Journal of Mathematical Biology, vol. 25, no. 4, pp. 359380, 1987.

[7] Y. Takeuchi, W. Ma, and E. Beretta, "Global asymptotic properties of a delay SIR epidemic model with finite incubation times," Nonlinear Analysis: Theory, Methods \& Applications, vol. 42, no. 6, pp. 931-947, 2000.

[8] E. Beretta, T. Hara, W. Ma, and Y. Takeuchi, "Global asymptotic stability of an SIR epidemic model with distributed time delay," Nonlinear Analysis, vol. 47, pp. 4107-4115, 2001.

[9] C. C. McCluskey, "Global stability of an SIR epidemic model with delay and general nonlinear incidence," Mathematical Biosciences and Engineering, vol. 7, no. 4, pp. 837-850, 2010.

[10] X. Zhou and J. Cui, "Stability and Hopf bifurcation of a delay eco-epidemiological model with nonlinear incidence rate," Mathematical Modelling and Analysis, vol. 15, no. 4, pp. 547-569, 2010.

[11] J.-J. Wang, J.-Z. Zhang, and Z. Jin, "Analysis of an SIR model with bilinear incidence rate," Nonlinear Analysis: Real World Applications, vol. 11, no. 4, pp. 2390-2402, 2010.

[12] Y. Enatsu, E. Messina, Y. Muroya, Y. Nakata, E. Russo, and A. Vecchio, "Stability analysis of delayed SIR epidemic models with a class of nonlinear incidence rates," Applied Mathematics and Computation, vol. 218, no. 9, pp. 5327-5336, 2012.

[13] S. Ruan and W. Wang, "Dynamical behavior of an epidemic model with a nonlinear incidence rate," Journal of Differential Equations, vol. 188, no. 1, pp. 135-163, 2003.

[14] W. Ma, M. Song, and Y. Takeuchi, "Global stability of an SIR epidemic model with time delay," Applied Mathematics Letters, vol. 17, no. 10, pp. 1141-1145, 2004.

[15] R. Xu and Z. Ma, "Global stability of a SIR epidemic model with nonlinear incidence rate and time delay," Nonlinear Analysis: Real World Applications, vol. 10, no. 5, pp. 3175-3189, 2009.

[16] C. W. Clark, Mathematical Bioeconomics: The Optimal Management of Renewable Resource, John Wiley \& Sons, New York, NY, USA, 2nd edition, 1990.

[17] P. W. Dong, S. Y. Zhuang, X. H. Lin, and X. Z. Zhang, "Economic evaluation of forestay industry based on ecosystem coupling," Mathematical and Computer Modelling, vol. 58, no. 6, pp. 10101017, 2013.

[18] N. Bairagi, S. Chaudhuri, and J. Chattopadhyay, "Harvesting as a disease control measure in an eco-epidemiological system-a theoretical study," Mathematical Biosciences, vol. 217, no. 2, pp. 134-144, 2009.

[19] S. Chakraborty, S. Pal, and N. Bairagi, "Dynamics of a ratiodependent eco-epidemiological system with prey harvesting," Nonlinear Analysis: Real World Applications, vol. 11, no. 3, pp. 1862-1877, 2010.
[20] R. Bhattacharyya and B. Mukhopadhyay, "On an eco-epidemiological model with prey harvesting and predator switching: local and global perspectives," Nonlinear Analysis: Real World Applications, vol. 11, no. 5, pp. 3824-3833, 2010.

[21] L. Zou, Z. Xiong, and Z. Shu, "The dynamics of an eco-epidemic model with distributed time delay and impulsive control strategy," Journal of the Franklin Institute, vol. 348, no. 9, pp. 2332-2349, 2011.

[22] H. S. Gordon, "The economic theory of a common property resource: the fishery," Journal of Political Economy, vol. 62, no. 2, pp. 124-142, 1954.

[23] C. Liu, Q. Zhang, Y. Zhang, and X. Duan, "Bifurcation and control in a differential-algebraic harvested prey-predator model with stage structure for predator," International Journal of Bifurcation and Chaos in Applied Sciences and Engineering, vol. 18, no. 10, pp. 3159-3168, 2008.

[24] C. Liu, Q. Zhang, X. Zhang, and X. Duan, "Dynamical behavior in a stage-structured differential-algebraic prey-predator model with discrete time delay and harvesting," Journal of Computational and Applied Mathematics, vol. 231, no. 2, pp. 612-625, 2009.

[25] X. Zhang, Q.-L. Zhang, C. Liu, and Z.-Y. Xiang, "Bifurcations of a singular prey-predator economic model with time delay and stage structure," Chaos, Solitons \& Fractals, vol. 42, no. 3, pp. 1485-1494, 2009.

[26] G. Zhang, B. Chen, L. Zhu, and Y. Shen, "Hopf bifurcation for a differential-algebraic biological economic system with time delay," Applied Mathematics and Computation, vol. 218, no. 15, pp. 7717-7726, 2012.

[27] K. Chakraborty, M. Chakraborty, and T. K. Kar, "Bifurcation and control of a bioeconomic model of a prey-predator system with a time delay," Nonlinear Analysis: Hybrid Systems, vol. 5, no. 4, pp. 613-625, 2011.

[28] C. Liu, Q. Zhang, J. Huang, and W. Tang, "Dynamical analysis and control in a delayed differential-algebraic bio-economic model with stage structure and diffusion," International Journal of Biomathematics, vol. 5, no. 2, pp. 1-30, 2012.

[29] S. Campbell, Singular Systems of Differential Equations, Priman, London, UK, 1980.

[30] P. Muller, "Linear mechanical descriptor systems: identification, analysis and design," in Proceedings of the IFAC International Conference on Control of Industrial Systems, pp. 501-506, Belfort, France, May 1997.

[31] M. S. Silva and T. P. de Lima, "Looking for nonnegative solutions of a Leontief dynamic model," Linear Algebra and its Applications, vol. 364, no. 1, pp. 281-316, 2003.

[32] S. Ayasun, C. O. Nwankpa, and H. G. Kwatny, "Computation of singular and singularity induced bifurcation points of differential-algebraic power system model," IEEE Transactions on Circuits and Systems I: Regular Papers, vol. 51, no. 8, pp. 1525$1538,2004$.

[33] M. Yue and R. Schlueter, "Bifurcation subsystem and its application in power system analysis," IEEE Transactions on Power Systems, vol. 19, no. 4, pp. 1885-1893, 2004.

[34] W. Marszalek and Z. W. Trzaska, "Singularity-induced bifurcations in electrical power systems," IEEE Transactions on Power Systems, vol. 20, no. 1, pp. 312-320, 2005.

[35] D. G. Luenberger, "Nonlinear descriptor systems," Journal of Economic Dynamics \& Control, vol. 1, no. 3, pp. 219-242, 1979. 
[36] D. G. Luenberger and A. Arbel, "Singular dynamic Leontief systems," Econometrics, vol. 45, no. 32, pp. 991-995, 1997.

[37] X. Yang, L. Chen, and J. Chen, "Permanence and positive periodic solution for the single-species nonautonomous delay diffusive models," Computers \& Mathematics with Applications, vol. 32, no. 4, pp. 109-116, 1996.

[38] H. R. Thieme, Mathematics in Population Biology, Princeton University Press, Princeton, NJ, USA, 2003.

[39] J. K. Hale and S. M. Verduyn Lunel, Introduction to FunctionalDifferential Equations, vol. 99, Springer, New York, NY, USA, 1993.

[40] Y. Kuang, Delay Differential Equations with Applications in Population Dynamics, vol. 191, Academic Press, New York, NY, USA, 1993.

[41] J. Guckenheimer and P. Holmes, Nonlinear Oscillations, Dynamical Systems, and Bifurcations of Vector Fields, vol. 42, Springer, New York, NY, USA, 1983.

[42] V. Venkatasubramanian, H. Schättler, and J. Zaborszky, "Local bifurcations and feasibility regions in differential-algebraic systems," IEEE Transactions on Automatic Control, vol. 40, no. 12, pp. 1992-2013, 1995.

[43] M. Kot, Elements of Mathematical Ecology, Cambridge University Press, Cambridge, UK, 2001.

[44] V. Venkatasubramanian, "Singularity induced bifurcation and the van der Pol oscillator," IEEE Transactions on Circuits and Systems I: Fundamental Theory and Applications, vol. 41, no. 11, pp. 765-769, 1994.

[45] R. E. Beardmore, "The singularity-induced bifurcation and its Kronecker normal form," SIAM Journal on Matrix Analysis and Applications, vol. 23, no. 1, pp. 126-137, 2001.

[46] L. Yang and Y. Tang, "An improved version of the singularityinduced bifurcation theorem," IEEE Transactions on Automatic Control, vol. 46, no. 9, pp. 1483-1486, 2001.

[47] L. Dai, Singular Control Systems, vol. 118, Springer, New York, NY, USA, 1989.

[48] H. I. Freedman and V. Sree Hari Rao, "The trade-off between mutual interference and time lags in predator-prey systems," Bulletin of Mathematical Biology, vol. 45, no. 6, pp. 991-1004, 1983.

[49] B. D. Hassard, N. D. Kazarinoff, and Y. H. Wan, Theory and Applications of Hopf Bifurcation, vol. 41, Cambridge University Press, Cambridge, UK, 1981. 


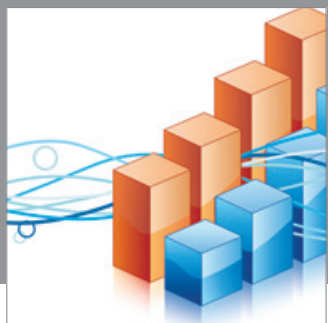

Advances in

Operations Research

mansans

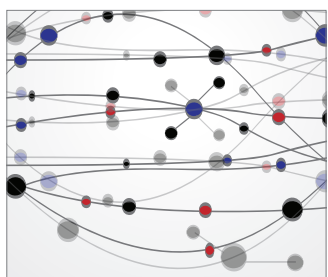

The Scientific World Journal
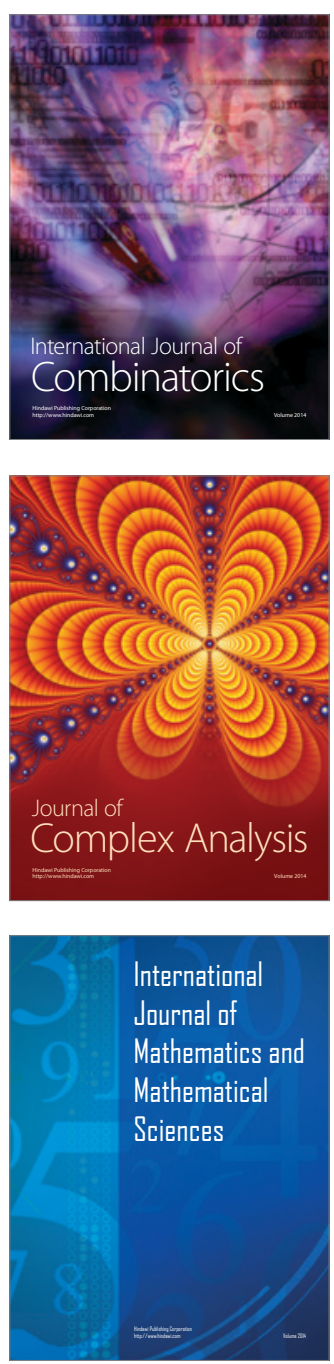
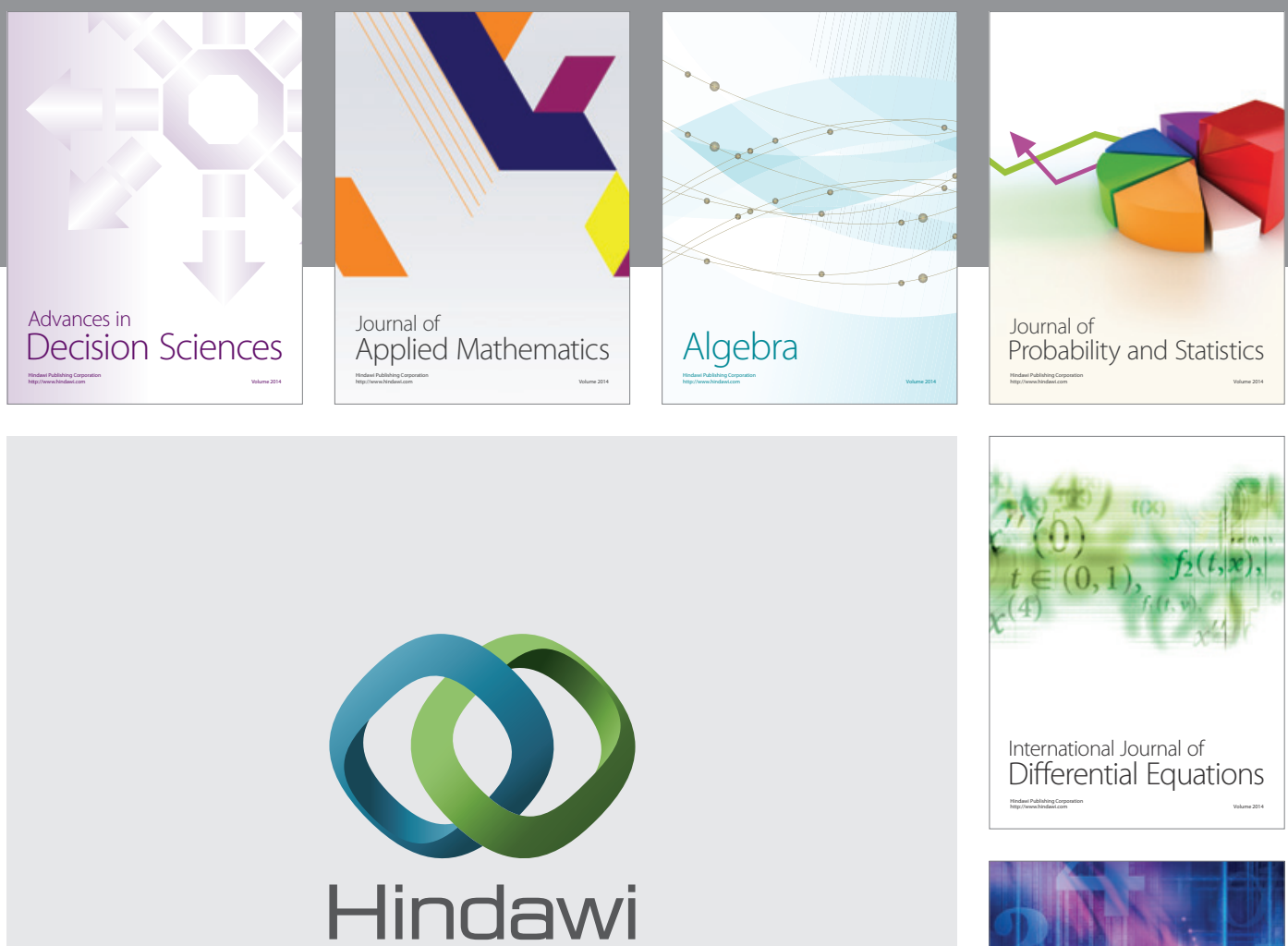

Submit your manuscripts at http://www.hindawi.com
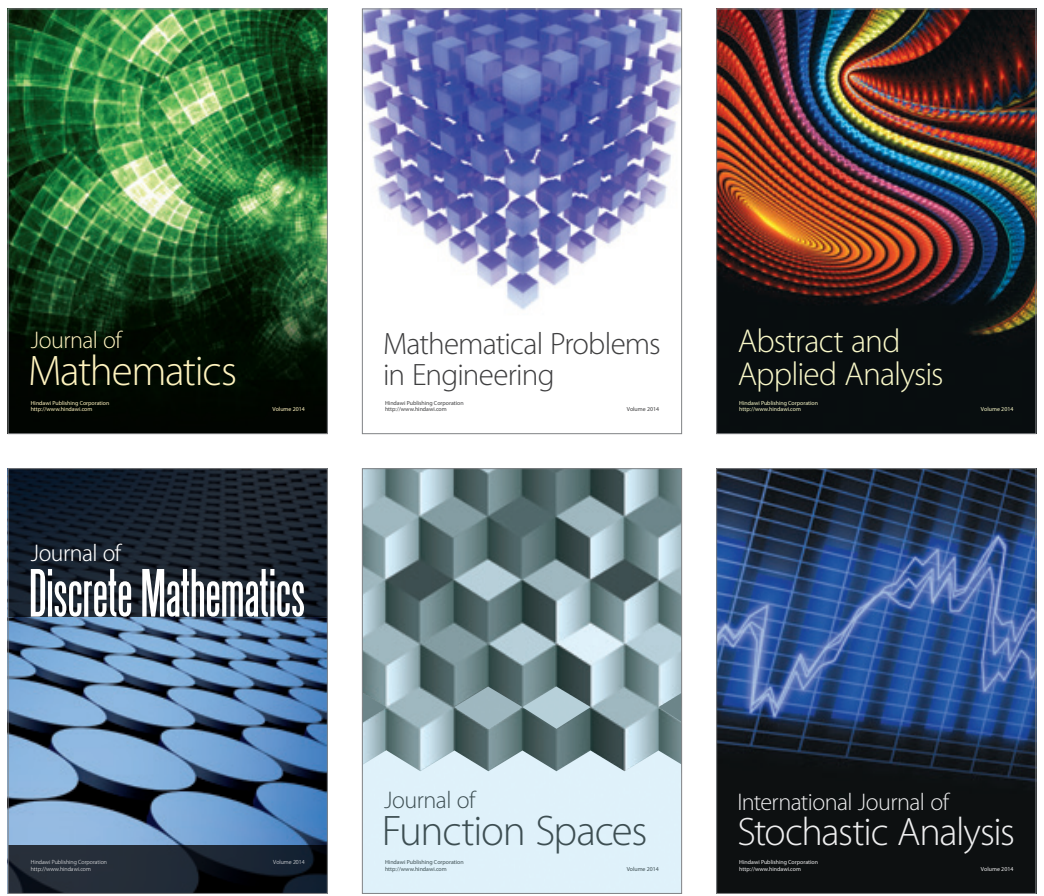

Journal of

Function Spaces

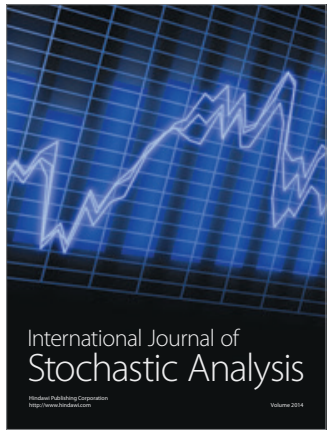

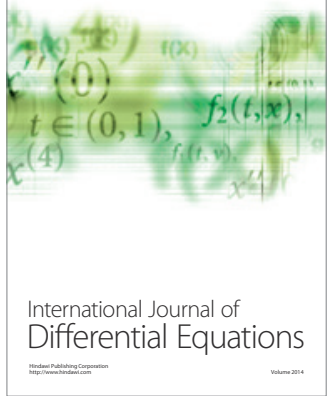
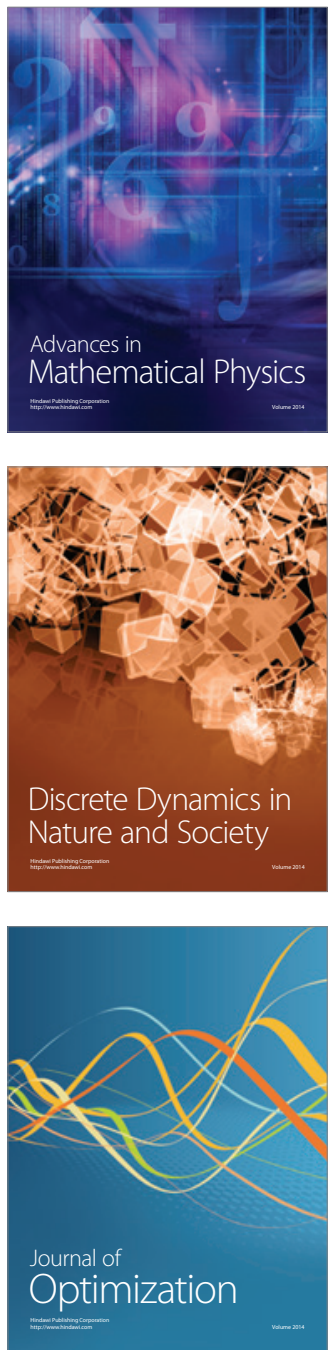